\title{
Electronic structure of Bas and boride III-V alloys
}

Gus L. W. Hart

gus.hart@gmail.com

Alex Zunger

Follow this and additional works at: https://scholarsarchive.byu.edu/facpub

Part of the Astrophysics and Astronomy Commons, and the Physics Commons

\section{Original Publication Citation}

G. L. W. Hart and A. Zunger, "BAs and Boride III-V alloys," Phys. Rev. B 62, 13522 (2). The original article may be found here: http://prb.aps.org/abstract/PRB/v62/i2/p13522_1

\section{BYU ScholarsArchive Citation}

Hart, Gus L. W. and Zunger, Alex, "Electronic structure of Bas and boride III-V alloys" (2000). Faculty Publications. 595.

https://scholarsarchive.byu.edu/facpub/595 


\title{
Electronic structure of BAs and boride III-V alloys
}

\author{
Gus L. W. Hart and Alex Zunger \\ National Renewable Energy Laboratory, Golden, Colorado 80401
}

(Received 16 June 2000)

\begin{abstract}
Boron arsenide, the typically ignored member of the Group-III-V arsenide series BAs-AlAs-GaAs-InAs is found to resemble silicon electronically: its $\Gamma$ conduction-band minimum is $p$-like $\left(\Gamma_{15}\right)$, not $s$-like $\left(\Gamma_{1 \mathrm{c}}\right)$, it has an $X_{1 \mathrm{c}}$-like indirect band gap, and its bond charge is distributed almost equally on the two atoms in the unit cell, exhibiting nearly perfect covalency. The reasons for these are tracked down to the anomalously low atomic $p$ orbital energy in the boron and to the unusually strong $s-s$ repulsion in BAs relative to most other Group-III-V compounds. We find unexpected valence-band offsets of BAs with respect to GaAs and AlAs. The valence-band maximum (VBM) of BAs is significantly higher than that of AlAs, despite the much smaller bond length of BAs, and the VBM of GaAs is only slightly higher than in BAs. These effects result from the unusually strong mixing of the cation and anion states at the VBM. For the BAs-GaAs alloys, we find (i) a relatively small $(\sim 3.5 \mathrm{eV})$ and composition-independent band-gap bowing. This means that while addition of small amounts of nitrogen to GaAs lowers the gap, addition of small amounts of boron to GaAs raises the gap; (ii) boron "semilocalized" states in the conduction band (similar to those in GaN-GaAs alloys); and (iii) bulk mixing enthalpies that are smaller than in GaN-GaAs alloys. The unique features of boride Group-III-V alloys offer new opportunities in band-gap engineering.
\end{abstract}

\section{INTRODUCTION}

With the advent of state-of-the-art techniques for growing semiconductor alloys on common substrates such as GaAs, silicon, and germanium, semiconductor compounds, which previously were very difficult to synthesize, are now routinely achieved. Techniques such as metal-organic chemical vapor deposition, molecular-beam epitaxy, and pulsed laser ablation have provided the opportunity to synthesize and study a large number of nitride, phosphide, and antimonide semiconductor alloys. Of particular recent interest are alloys of a wide gap semiconductor (e.g., nitrides) with a "conventional" Group-III-V semiconductor because of their promise in optical applications. Two diverging scenarios were considered: (i) using a significant amount (10\%-30\%) of the wide gap component to shift the alloy band-gap to the blue (e.g., adding $\sim 20 \% \mathrm{GaN}$ to $\mathrm{InN}$ ) for light-emitting diode or laser applications, and (ii) using a small amount of the wide gap semiconductor to shift the alloy band-gap to the red (e.g., adding 1\%-3\% GaN to GaAs) for photovoltaic applications. The latter effect occurs naturally if the band-gap bowing parameter $b$ is larger than the difference of the band gaps of the constituents (e.g., ZnS-ZnTe; GaAs-GaN). In this case addition of small amounts of the wide gap components acts to initially lower the band gap of the small gap component. For example, one can achieve the technologically desired 1-eV gap if one adds nitrogen to GaAs or to InGaAs.

When boron is substituted into GaAs, it can go to either a gallium site or an arsenic site. Normally boron prefers isovalent substitution on the gallium site,${ }^{1-3}$ which is the case we study here. In the other case when boron goes to the arsenic site (a boron "antisite" defect), the boron acts as an acceptor and this antisite defect has been the subject of numerous studies. ${ }^{4-8}$ Growth conditions determine whether boron goes to the gallium site as an isovalent substitution or to the arsenic site as an acceptor. For example, $\mathrm{B}_{\mathrm{As}}$ antisite defects are known to occur in Ga-rich samples of GaAs grown by the liquid encapsulated Czochralski method, but for GaAs crystals taken from stoichiometric or As-rich melts, electrically active boron or boron complexes are not found, ${ }^{9}$ indicating that the boron atoms have substituted isovalently to the gallium sites. Not as much is known about the epitaxial growth conditions under which isovalent or antisite boron incorporation occurs. It is reasonable to suppose that $\mathrm{B}_{\mathrm{As}}$ antisite defects will be more likely under Ga-rich conditions and that As-rich growth conditions will lead to isovalent boron incorporation, similar to the case for LEC-grown GaAs. In this paper, we focus on isovalent $\mathrm{BGaAs}$ alloys where boron occupies gallium sites.

In this paper we will explore BAs as an alternative to GaN as a wide gap partner for alloying conventional GroupIII-V's such as AlAs and GaAs. Very little is known about BAs: As early as 1966, Ku mentioned the possibilities of BAs-GaAs as a boride Group-III-V alloy with potentially useful properties. ${ }^{10,11}$ However, difficulties in fabricating BAs or simple solid solutions of zincblende BAs-GaAs (Refs. 10,12-15) prior to the development of current epitaxial techniques have prevented a careful study of the alloy properties. Now that epitaxial techiques have eased these difficulties somewhat, boride semiconductor alloys are generating renewed interest. ${ }^{7,8,16-18}$ There are a number of theoretical studies that examine different aspects of pure BAs ${ }^{19-26}$ but theoretical studies of the boron alloys are lacking.

Important questions that one would want to answer include:

(i) How will alloying with BAs affect the band-gaps and other properties of GaAs or AlAs?

(ii) To what extent does BAs fit the well-known trends in band gaps, band offsets, and bonding patterns of the arsenide Group-III-V series BAs-AlAs-GaAs-InAs?

(iii) Will the band-gap bowing of $\mathrm{B}_{x} \mathrm{Ga}_{1-x} \mathrm{As}$ alloys be as 
anomalous as the (very large and composition dependent) bowing in $\mathrm{GaAs}_{1-x} \mathrm{~N}_{x}$ ?

(iv) Will cation substitution by boron lead to unusual wave function localization effects found to exist for anion substitition by nitrogen $?^{27-29}$

Two features of boron make boride semiconductors fundamentally different from common Group-III-V or GroupII-VI semiconductors. The first is that, like nitrogen, boron is in the first row of the Periodic Table and has deep $p$ orbitals and a small atomic size. The second feature is, unlike nitrogen, boron has a low electronegativity. This leads to highly covalent compounds, unlike nitride semiconductors, which have a strong ionic character. This paper examines (i) zincblende BAs and its place in the Group-III-As family of semiconductors and (ii) boron substitution of gallium in GaAs, including alloy bowing, band offsets, and mixing enthalpies. Our main findings are:

Zincblende BAs: Surprisingly, we find that, electronically, BAs resembles silicon rather than other Group-III-V semiconductors. Similar to silicon and in contrast to most GroupIII-V's, the lowest Brillouin-zone center conduction band of BAs has $p$ symmetry $\left(\Gamma_{15 \mathrm{c}}\right)$ rather than $s$ symmetry $\left(\Gamma_{1 \mathrm{c}}\right)$, and, like silicon, its total valence charge density shows almost symmetric distribution of charge around the two atoms in the unit cell. The reasons for the siliconlike conductionband ordering in BAs are: (i) the small repulsion of the bonding and antibonding $p$ states due to the low $p$ orbital energy of boron, as well as the unusual hybridization of both cation and anion $p$ states at the VBM, and (ii) the repulsion of the cation and anion $s$ states that is much stronger in BAs than in AlAs, GaAs, and InAs. As a result of the $p$ - $p$ hybridization (covalent bonding) mentioned in (i), we also find that the valence-band offset of BAs relative to other members of the Group-III-As family is unusually high.

BAs-GaAs alloys: (i) The band-gap bowing is relatively small $(\sim 3.5 \mathrm{eV})$ and composition independent, in stark contrast to GaN-GaAs alloys. Because of this small bowing, the addition of BAs to GaAs increases the gap, thus, unlike nitrogen, addition of boron into GaAs or InGaAs will not lead to the desired $1 \mathrm{eV}$ material. (ii) The lower energy conduction-band states are "semilocalized" states around the boron atoms, e.g., the conduction-band maximum (CBM) is strongly localized near the boron but extended at longer distances while the VBM is completely delocalized. (iii) The bulk mixing enthalpy of BAs in GaAs is much lower than that of $\mathrm{GaN}$ in GaAs, indicating that the bulk solubility of boron in Group-III-V compounds may be higher than that of nitrogen and thus higher composition ranges may be possible with the boride alloys. These findings indicate that boride Group-III-V alloys provide new opportunities in band-gap engineering.

\section{METHODS OF CALCULATION}

\section{A. The LAPW calculations}

We used density-functional theory within the localdensity approximation (LDA) ${ }^{30}$ as implemented by the fullpotential linearized-augmented-plane waves (LAPW) method $^{31,32}$ (WIEN97 implementation ${ }^{33}$ ). The exchangecorrelation potential of Perdew and Wang was used. ${ }^{34}$ In the calculations with less than 32 atoms, the plane-wave kinetic- energy cutoff for the expansion in the interstitial region was 16 Ry (approximately 130 basis functions per atom). The muffin-tin (MT) radii were $1.65 \mathrm{bohr}$ for boron and $2.2 \mathrm{bohr}$ for arsenic, aluminum, gallium, and indium. In the large supercell calculations ( 32 or more atoms), a slightly smaller plane-wave kinetic-energy cutoff of $13 \mathrm{Ry}$ (approximately 120 basis functions per atom) and a larger boron MT radius of $1.8 \mathrm{bohr}$ ( 2.1 for arsenic, aluminum, gallium, and indium) was used to ease the computational burden of the larger cells. Our convergence studies indicate that the error in the individual eigenstates is less than $5 \mathrm{meV}$ for the valence and lower-conduction bands. The calculations were run until the variation in the total energy between several self-consistency cycles was $<10^{-5} \mathrm{Ry}$. The experimental lattice constants were used in all the calculations of the individual compounds. The experimental lattice constants are 4.777, 5.660, 5.653, and $6.058 \AA$ for BAs, AlAs, GaAs, and InAs, respectively.

The $k$-point mesh used in the calculation of the simple binary compounds (zincblende BAs, AlAs, GaAs, and InAs) was a $4 \times 4 \times 4$ mesh of Monkhorst and Pack special points [10 points in the irreducible wedge of the Brillouin zone (BZ).$^{35}$ The superlattice calculations for the valence-band offsets as well as the supercell calculations for the alloy studies used $k$-point meshes equivalent ${ }^{36}$ to the $4 \times 4 \times 4$ mesh used in the calculation of the simple zincblende binary compounds. Using equivalent $k$-point meshes is particularly important for calculations such as enthalpies of formation in order to eliminate uncertainties due to the statistical errors of different $k$-point meshes. Thus, one can use much smaller $k$-point meshes to achieve the required accuracies than would otherwise be necessary.

\section{B. Partial DOS, band characters, and valence charge density}

It is useful to analyze the orbital character of different states. The band character (or orbital population) $Q_{l}^{(\alpha)}(\epsilon, \mathbf{k})$ is the $l$ th angular momentum component of the charge due to wave function $\psi(\epsilon, \mathbf{k})$ enclosed in a sphere $\Omega_{\mathrm{MT}}$ of radius $R_{\mathrm{MT}}^{(\alpha)}$ about atom $\alpha$ :

$$
Q_{l}^{(\alpha)}(\epsilon, \mathbf{k})=\int_{\Omega_{\mathrm{MT}}}\left|\hat{P}_{l} \psi(\epsilon, \mathbf{k}, \mathbf{r})\right|^{2} d \mathbf{r},
$$

where $\hat{P}_{l}$ is an angular momemtum projection operator with origin at site $\alpha$. Because the interstitial region outside the muffin-tin spheres is excluded when the band characters are calculated, the muffin-tin radii were chosen to match the rationalized tetrahedral radii of Phillips. ${ }^{37}$

The partial density-of-states (DOS) are determined by integrating $Q_{l}^{(\alpha)}(\epsilon, \mathbf{k})$ over all $\mathbf{k}$ in the BZ:

$$
N_{l}^{(\alpha)}(\epsilon)=\int_{\mathrm{BZ}} Q_{l}^{(\alpha)}(\epsilon, \mathbf{k}) d \mathbf{k} .
$$

The angular momentum decomposition of the total valence charge can then be determined by summing $N_{l}(\epsilon)$ over the valence bands:

$$
q_{l}^{\mathrm{tot}}=\int_{\mathrm{VB}_{\min }}^{\mathrm{VB}_{\max }} N_{l}(\epsilon) d \epsilon .
$$


TABLE I. Convergence test for the valence-band offsets of BAs/GaAs, BAs/AlAs, and AlAs/GaAs using two different superlattice periods. Units are $\mathrm{eV}$. Band offsets were calculated using both anion and cation core states [see Eq. (6)]. Note that while for AlAs/GaAs a similar offset is obtained when either anion or cation core levels are used, for the borides only anion core levels provide a rapidly convergent band offset with respect to the superlattice period $n$.

\begin{tabular}{cccc}
\hline \hline Superlattice & $\begin{array}{c}\text { Using } \\
1 s \text { anion }\end{array}$ & $\begin{array}{c}\text { Using } \\
2 s \text { anion }\end{array}$ & $\begin{array}{c}\text { Using } \\
1 s \text { cation }\end{array}$ \\
\hline$(\mathrm{BAs})_{2} /(\mathrm{GaAs})_{2}$ & 0.18 & 0.11 & 0.83 \\
$(\mathrm{BAs})_{4} /(\mathrm{GaAs})_{4}$ & 0.19 & 0.12 & 0.58 \\
$(\mathrm{BAs})_{2} /(\mathrm{AlAs})_{2}$ & -0.38 & -0.45 & 0.58 \\
$(\mathrm{BAs})_{4} /(\mathrm{AlAs})_{4}$ & -0.39 & -0.47 & 0.17 \\
$(\mathrm{AlAs})_{2} /(\mathrm{GaAs})_{2}$ & 0.50 & 0.50 & 0.41 \\
$(\mathrm{AlAs})_{4} /(\mathrm{GaAs})_{4}$ & 0.51 & 0.51 & 0.47 \\
\hline \hline
\end{tabular}

Valence charge densities: The charge density is constructed from the highest $N_{B}$ occupied bands as

$$
\rho_{\mathrm{val}}(\mathbf{r})=\sum_{n=1}^{N_{B}} \int_{\mathrm{BZ}}\left|\psi_{n, \mathbf{k}}(\mathbf{r})\right|^{2} d \mathbf{k} .
$$

For plotting $\rho_{\text {val }}$ we use $N_{B}=4$. Thus, cation $d$ bands are not included in the construction.

The "valence deformation density" $\Delta \rho_{\text {val }}(\mathbf{r})$ describes the difference between the solid-state density and a superposition of densities of spherical atoms in their ground state,

$$
\Delta \rho_{\mathrm{val}}(\mathbf{r})=\rho_{\mathrm{val}}(\mathbf{r})-\rho_{\text {sup }}(\mathbf{r}) .
$$

The valence charge difference is defined as the difference between the crystal valence density (including the cation $d$ bands, when occupied) and a superposition of free, spherical LDA atomic densities arranged in the configuration of the crystal. The atomic configuration of the free atoms are $d^{10} s^{2} p^{1}$ for the cations and $s^{2} p^{3}$ for the anions.

\section{Band offsets}

The offset $\Delta E_{v}(A X / B Y)$ between the valence-band maxima of two semiconductor compounds $A X$ and $B Y$ forming a heterostructure is calculated using a method similar to that used in photoemission spectroscopy. ${ }^{38}$

$$
E_{v}(A X / B Y)=\Delta E_{v, C^{\prime}}^{B Y}-\Delta E_{v, C}^{A X}+\Delta E_{C, C^{\prime}}^{A X / B Y},
$$

where

$$
\begin{aligned}
& \Delta E_{v, C}^{A X}=E_{v}^{A X}-E_{C}^{A X}, \\
& \Delta E_{v, C^{\prime}}^{B Y}=E_{v}^{B Y}-E_{C^{\prime}}^{A X}
\end{aligned}
$$

are the energy seperations between the core levels ( $C$ and $C^{\prime}$ ) and the valence-band maximums for the pure $A X$ and $B Y$ compounds. The third term in Eq. (6),

$$
\Delta E_{C, C^{\prime}}^{A X / B Y}=E_{C^{\prime}}^{B Y}-E_{C}^{A X}
$$

is the difference in the core-level binding energy between the two compounds $A X$ and $B Y$ in the $A X / B Y$ heterojunction. To calculate the "natural band offset," $\Delta E_{v, C}^{A X}$ and $\Delta E_{v, C^{\prime}}^{B Y}$ are calculated for $A X$ and $B Y$ at their cubic, equilibrium lattice constants. The core-level difference $\Delta E_{C, C^{\prime}}^{A X / B Y}$ is ob- tained from calculations of $(A X)_{n} /(B Y)_{n}$ superlattices in the (001) direction. The period $n$ needs to be large enough so that $A X$-like and $B Y$-like properties can be identified far from the interface. For the core states (denoted $C$ and $C^{\prime}$ ), we use the anion $1 s$ states. Table I shows the dependence of the valence-band offset calculations on the period of the superlattice and on which core states are used. Because of the large size mismatch of BAs and GaAs, at least $n=4$ is required to sufficiently converge $\Delta E_{C, C^{\prime}}^{A X / B Y}$. We also find that while a similar offset is obtained for AlAs/GaAs, when either anion or cation core levels are used, for the borides only, anion core levels provide a rapidly convergent band offset with respect to the superlattice period $n$.

\section{Alloy enthalpies of mixing, alloy bowing, and equivalent $k$ points}

The enthalpy of mixing for an alloy $A_{1-x} B_{x}$ of two components $A$ and $B$ is the difference in energy between the alloy and the weighted sum of the constituents:

$$
\Delta H(x)=E_{A_{p} B_{q}}-\left[(1-x) E_{A}+x E_{B}\right],
$$

where $x=p /(p+q)$. In the mixing enthalpy calculations, the alloy lattice constant $a(x)$ is taken as the linear average of the experimental values of the constituents. The experimental lattice constants of BAs and GaAs are 4.777 and $5.653 \AA$, respectively, whereas the calculated lattice constants are 4.740 and $5.615 \AA$. Any free internal coordinates in the alloy structure were relaxed using the quantummechanical forces so that residual forces on the ions were less than $1 \mathrm{mRy} / \mathrm{bohr}$.

The band-gap bowing parameter $b$ defined by

$$
E_{g}(x)=\bar{E}_{g}(x)-b x(x-1),
$$

where $\bar{E}_{g}(x)$ is the weighted linear average of the individual band-gaps of $A$ and $B$. The bowing parameter $b$ represents the deviation of the band-gap from this average. Note that although there are LDA errors in the calculated gaps of the $A_{x} B_{1-x}$ alloy as well as the pure constituents $\mathrm{A}$ and $\mathrm{B}$, to lowest order, the LDA error in the bowing $b$ cancels out.

Considering Eqs. (9) and (10), we see that one needs to converge the $k$ representation for a compound $A_{p} B_{q}$ as well as for the elemental constituents $A$ and $B$. The standard way 
of accomplishing this is to increase the number of $k$ points in all three systems until convergence is obtained. The disadvantage of this approach is that it requires absolute $k$-point convergence for $A$ and $B$, and separately for $A_{p} B_{q}$. A better approach is to take advantage of relative $k$-point convergence. ${ }^{36}$ The idea is to sample the Brillouin zone equivalently for $A, B$ and $A_{p} B_{q}$. This could be done by considering $A_{p} A_{q}, B_{p} B_{q}$, and $A_{p} B_{q}$ as isostructural solids and sampling the Brillouin zone of each equally. Then, any relative $k$-point sampling error cancels out. This is called the method of equivalent $k$ points. ${ }^{36}$ In practice, one does not have to calculate $A_{p} A_{q}$ and $B_{p} B_{q}$ but instead can calculate $A$ and $B$ in their primitive unit cells using suitably folded-in $k$ points. Equivalent $k$ points for the unit cells in this paper are given in Table II.

\section{E. Choice of supercells}

The calculations of $\Delta H$ [Eq. (9)] and $b$ [Eq. (10)] require supercells. We use $\mathrm{B}_{1} \mathrm{Ga}_{7} \mathrm{As}_{8}, \mathrm{~B}_{1} \mathrm{Ga}_{15} \mathrm{As}_{16}, \mathrm{~B}_{1} \mathrm{Ga}_{31} \mathrm{As}_{32}$, and $\mathrm{B}_{2} \mathrm{Ga}_{30} \mathrm{As}_{32}$. The lattice vectors defining the supercells are given in Table II. The SQS16 supercell is a "special quasirandom structure" - a periodic structure with a rather small unit cell whose lattice sites are occupied by $A$ and $B$ atoms so as to mimic the atom-atom correlation functions of much larger $A_{1-x} B_{x}$ supercells with random occupations. ${ }^{39}$

In the calculations for the band-gap bowing of $\mathrm{B}_{x} \mathrm{Ga}_{1-x} \mathrm{As}$ alloys, 64 atom, simple-cubic unit cells were used for both the $3 \%$ and $6 \%$ boron alloys. In the case of the $3 \%$ alloy, there is one boron atom in the supercell, but for the $6 \%$ alloy, there are two boron atoms in the supercell. For this case, the band-gap was determined by taking the weighted average of the gaps for the 5 symmetrically inequivalent configurations (given in Table III) of two boron atoms in the 64-atom supercell. These five pairs are the first through fourth neighbor pairs in an fcc lattice, as well as the sixth neighbor. (In the 64-atom cell, the fifth fcc neighbor is equivalent to the first.) Using the same 64-atom unit cell for the alloys in the bowing calculations eliminated band-gap differences that can occur due to the $k$-point folding relations of different supercells, as discussed by Bellaiche et al. in Ref. 28.

\section{F. Valence force field model}

In two cases, the total energies or the internal structural parameters were determined using a classical force field model. This generalized valence force field model ${ }^{40,41}$ (VFF) consists of bond-stretching, bond-bending, and bendingstretching springs with force constants $\sigma, \beta$, and $\sigma$, respectively. The force constants and the equilibrium (no force) bond lengths are given in Table IV. The two cases where the VFF model was used are:

(1) The VFF model was used to model the mixing enthalpies of boron or nitrogen in GaAs in the regime of very low concentrations (Sec. V C). Because well-tested force constants exist for the "conventional" VFF model $(\alpha, \beta$; no $\sigma)$ for the case of $\mathrm{GaAs} / \mathrm{GaN}$, we also used a two-parameter $(\sigma=0)$ model for BAs/GaAs for the sake of consistency with previous calculations. The parameters for BAs/GaAs were tested by comparing the enthalpies of formation for structures at higher concentrations calculated using first principles, as given in Table $\mathrm{V}$.

(2) Full relaxation using first-principles' forces of all 64atom supercells used in the band-gap bowing calculations reported in Sec. V B is computationally prohibitive so the generalized $^{41}$ VFF model was used to determine internal coordinates. In one case $\left(\mathrm{B}_{1} \mathrm{Ga}_{31} \mathrm{As}_{32}\right)$, the VFF- and LAPWdetermined coordinates were compared and found to be very similar. The average difference between the two methods for the Ga-As bonds was $<0.005$ a.u. $(0.1 \%)$. The largest discrepency was near the boron site where the VFF predicts a B-As bond length that is 0.01 a.u. (1.5\%) shorter than the LAPW bond length. Using the VFF-determined internal coordinates of the supercell would be acceptably accurate only if the band-gap is very close to that when the first-principles' coordinates are used. In the case of the $\mathrm{BGa}_{31} \mathrm{As}_{32}$ supercell just mentioned, the band-gap difference was only $10 \mathrm{meV}$, justifying the use of the VFF.

\section{BAs AND THE III-As FAMILY}

\section{A. Expectations from atomic physics}

The LDA valence orbital energies for the first four elements of column III are plotted in Fig. 1. The lower value of the atomic $p$ states of $\mathrm{B}$ relative to the atomic $p$ states for $\mathrm{Al}$, $\mathrm{Ga}$, and In (which are all quite similar) are notable. This lower value can be understood from the fact that, as a first row element, the $2 p$ states in B need not be orthogonal to lower $p$ states. In general, the cation $s$ energy should increase going down the column but there is a kink when moving from $\mathrm{Al}$ to $\mathrm{Ga}$ due to the introduction of imperfectly screened $d$ states in $\mathrm{Ga}$. The fact that the $p$ states in $\mathrm{B}$ lie lower in energy, and hence closer to the As $4 p$ states, will lead to a much stronger hybridization of $p$-like states in BAs relative to the other Group-III-As systems.

The consequence of this is shown in Fig. 2 which shows the total valence charge, $q_{l}^{\text {tot }}$ of angular momentum $l[\mathrm{Eq}$. (3)], enclosed in spheres having the tetrahedral radii of Phillips. ${ }^{37}$ It is clear that the occupation of the B and As $p$-like states in BAs is much higher than in the other systems (indicating that $p-p$ hybridization is much more pronounced in BAs) and also that there is more charge around the boron than the other cations. Thus, the distinction between "cation" and "anion" is not so clear cut in BAs, where both atoms share similar charge. This is also reflected in the relative ionicities in the group. According to the Phillips scale ${ }^{42}$ the relative ionicities are $0.002,0.274,0.310$, and 0.357 for BAs, AlAs, GaAs, and InAs, respectively. As a result of the relative ionicities, the bonding in BAs is almost completely covalent whereas the other three members of the group have a significant ionic component that increases in the order $\mathrm{AlAs} \rightarrow \mathrm{GaAs} \rightarrow \mathrm{InAs}$. We will see next how these expectations are reflected in the electronic structure of BAs.

\section{B. Energy bands, band characters, and densities-of-states in zincblende BAs}

The energy bands of zincblende BAs are shown in Fig. 3. Calculated band energies at high-symmetry points are given in Table VI. ${ }^{34,24}$ As others have shown previously, ${ }^{13,24,19,23}$ 
TABLE II. Definition of the supercells used in this study and equivalent $k$ points. Lattice vectors are given in units of $a_{0}$ and equivalent $k$ points are given as fractions of the reciprocal lattice vectors.

\begin{tabular}{|c|c|c|c|}
\hline System & Lattice vectors & Equivalent $k$ points & Relative weight \\
\hline \multirow[t]{10}{*}{$A C, B C$ zincblende } & $(1 / 2,1 / 2,0)$ & $(0,0,1 / 8)$ & 1 \\
\hline & $(1 / 2,0,1 / 2)$ & $(0,0,3 / 8)$ & 1 \\
\hline & $(0,1 / 2,1 / 2)$ & $(0,1 / 8,3 / 4)$ & 3 \\
\hline & & $(0,1 / 8,1 / 4)$ & 3 \\
\hline & & $(0,1 / 8,1 / 2)$ & 3 \\
\hline & & $(0,1 / 4,5 / 8)$ & 3 \\
\hline & & $(1 / 8,1 / 4,1 / 2)$ & 6 \\
\hline & & $(0,1 / 4,3 / 8)$ & 3 \\
\hline & & $(0,3 / 8,1 / 2)$ & 3 \\
\hline & & $(1 / 8,3 / 8,5 / 8)$ & 6 \\
\hline \multirow{12}{*}{$(A C)_{1} /(B C)_{1}(100)$ superlattice } & $(1 / 2,1 / 2,0)$ & $(0,1 / 8,1 / 8)$ & 1 \\
\hline & $(1 / 2,-1 / 2,0)$ & $(0,1 / 8,3 / 8)$ & 1 \\
\hline & $(0,0,1)$ & $(0,3 / 8,1 / 8)$ & 1 \\
\hline & & $(0,3 / 8,3 / 8)$ & 1 \\
\hline & & $(1 / 8,1 / 4,1 / 8)$ & 2 \\
\hline & & $(1 / 8,1 / 4,3 / 8)$ & 2 \\
\hline & & $(1 / 8,1 / 2,1 / 8)$ & 1 \\
\hline & & $(1 / 8,1 / 2,3 / 8)$ & 1 \\
\hline & & $(1 / 4,3 / 8,1 / 8)$ & 2 \\
\hline & & $(1 / 4,3 / 8,3 / 8)$ & 2 \\
\hline & & $(3 / 8,1 / 2,1 / 8)$ & 1 \\
\hline & & $(3 / 8,1 / 2,3 / 8)$ & 1 \\
\hline \multirow[t]{6}{*}{$(A C)_{2} /(B C)_{2}(100)$ superlattice } & $(1 / 2,1 / 2,0)$ & $(0,1 / 8,1 / 4)$ & 1 \\
\hline & $(1 / 2,-1 / 2,0)$ & $(0,3 / 8,1 / 4)$ & 1 \\
\hline & $(0,0,2)$ & $(1 / 8,1 / 4,1 / 4)$ & 2 \\
\hline & & $(1 / 8,1 / 2,1 / 4)$ & 1 \\
\hline & & $(1 / 4,3 / 8,1 / 4)$ & 2 \\
\hline & & $(3 / 8,1 / 2,1 / 4)$ & 1 \\
\hline \multirow[t]{6}{*}{$(A C)_{4} /(B C)_{4}(100)$ superlattice } & $(1 / 2,1 / 2,0)$ & $(0,1 / 8,1 / 2)$ & 1 \\
\hline & $(1 / 2,-1 / 2,0)$ & $(0,3 / 8,1 / 2)$ & 1 \\
\hline & $(0,0,4)$ & $(1 / 8,1 / 4,1 / 2)$ & 2 \\
\hline & & $(1 / 8,1 / 2,1 / 2)$ & 1 \\
\hline & & $(1 / 4,3 / 8,1 / 2)$ & 2 \\
\hline & & $(3 / 8,1 / 2,1 / 2)$ & 1 \\
\hline \multirow[t]{3}{*}{$A_{1} B_{7} C_{8}$ fcc supercell } & $(1,1,0)$ & $(0,0,1 / 4)$ & 1 \\
\hline & $(1,0,1)$ & $(0,1 / 4,1 / 2)$ & 3 \\
\hline & $(0,1,1)$ & & \\
\hline \multirow[t]{3}{*}{$A_{1} B_{15} C_{16}$ bcc supercell } & $(1,1,-1)$ & $(1 / 8,1 / 8,1 / 8)$ & 1 \\
\hline & $(1,-1,1)$ & $(1 / 8,5 / 8,5 / 8)$ & 1 \\
\hline & $(-1,1,1)$ & & \\
\hline \multirow[t]{3}{*}{$A_{1} B_{31} C_{32}$ simple cubic supercell } & $(2,0,0)$ & $(1 / 4,1 / 4,1 / 4)$ & 1 \\
\hline & $(0,2,0)$ & & \\
\hline & $(0,0,2)$ & & \\
\hline \multirow[t]{8}{*}{$A_{8} B_{8} C_{16} \mathrm{SQS} 16$} & $(1,-1,2)$ & $(0,1 / 2,0)$ & 1 \\
\hline & $(1,-1-2)$ & $(1 / 2,0,0)$ & 1 \\
\hline & $(1 / 2,1 / 2,0)$ & $(1 / 4,3 / 4,1 / 8)$ & 4 \\
\hline & & $(0,1 / 2,1 / 4)$ & 2 \\
\hline & & $(1 / 2,0,1 / 4)$ & 2 \\
\hline & & $(1 / 4,3 / 4,3 / 8)$ & 4 \\
\hline & & $(0,1 / 2,1 / 2)$ & 1 \\
\hline & & $(1 / 2,0,1 / 2)$ & 1 \\
\hline
\end{tabular}


TABLE III. All possible pair configurations in the 64-atom cell.

\begin{tabular}{ccc}
\hline \hline $\begin{array}{c}\text { Shell number } \\
(n \text {th fcc neighbor })\end{array}$ & $\begin{array}{c}\text { Lattice vector } \\
{[u, v, w]\left(\text { unit } a_{0} / 2\right)}\end{array}$ & $\begin{array}{c}\text { Number of } \\
\text { equivalent pairs }\end{array}$ \\
\hline 1 & {$[0,1,1]$} & 12 \\
2 & {$[2,0,0]$} & 3 \\
3 & {$[2,1,1]$} & 12 \\
4 & {$[2,2,0]$} & 3 \\
6 & {$[2,2,2]$} & 1 \\
\hline \hline
\end{tabular}

the band gap of BAs is indirect; the CBM is along the $\Delta$ line between the $\Gamma$ and $X$ points-at approximately $0.82(1,0,0) 2 \pi / a$. An unusual feature of the band structure is the character of the CBM at $\Gamma$. In BAs, the CBM is the $p$-like $\Gamma_{7 \mathrm{c}}$ state $\left(\Gamma_{15 \mathrm{c}}\right.$ if spin-orbit interaction not included $)$. Only the semiconductors silicon and BP share this feature. In most semiconductors, the lowest state at $\Gamma$ is the singly degenerate $s$-like state. The origins of this feature of the BAs band structure can be understood in the context of the tight-binding model of Harrison. ${ }^{43}$ According to this model, the bonding $\left(\Gamma_{15 \mathrm{v}}\right)$ and antibonding $\left(\Gamma_{15 \mathrm{c}}\right) p$ states at $\Gamma$ are given by

$$
E\left(\Gamma_{15}\right)=\frac{\varepsilon_{p}^{c}+\varepsilon_{p}^{a}}{2} \pm \sqrt{\left(\frac{\varepsilon_{p}^{c}-\varepsilon_{p}^{a}}{2}\right)^{2}+\left(4 E_{p p}\right)^{2}}
$$

where $\varepsilon_{p}^{c}$ and $\varepsilon_{p}^{a}$ are the $p$ atomic orbital energies for the cation $(c)$ and the anion $(a)$ and the interatomic term $E_{p p}$ is proportional to $1 / d_{\text {bond }}^{2}$ where $d_{\text {bond }}$ is the bond length. The bonding $\left(\Gamma_{1 \mathrm{v}}\right)$ and antibonding $\left(\Gamma_{1 \mathrm{c}}\right) s$ states at $\Gamma$ are given by

$$
E\left(\Gamma_{1}\right)=\frac{\varepsilon_{s}^{c}+\varepsilon_{s}^{a}}{2} \pm \sqrt{\left(\frac{\varepsilon_{s}^{c}-\varepsilon_{s}^{a}}{2}\right)^{2}+\left(4 E_{s s}\right)^{2}}
$$

where $\varepsilon_{s}^{c}$ and $\varepsilon_{s}^{a}$ are the $s$ atomic orbital energies for the cation $(c)$ and the anion $(a)$ and the interatomic term $E_{s s}$ is again proportional to $1 / d_{\text {bond }}^{2}$. A schematic diagram of the BAs energy levels in this model is shown in Fig. 4(a).

The reversal of the conduction-band states in the BAs band structure relative to other Group-III-V compounds is a result of two effects: (i) a small $\Gamma_{15 \mathrm{v}}-\Gamma_{15 \mathrm{c}}$ bonding/ antibonding repulsion due to the small $E_{p p}$, as described by Eq. (11), and (ii) a large $\Gamma_{1 \mathrm{v}}-\Gamma_{1 \mathrm{c}}$ repulsion due to the small

TABLE IV. Parameters used in the VFF model. $d_{\text {bond }}^{0}$ is the equilibrium (unstretched) bond length, $\alpha$ is the bond-stretch term, $\beta$ is the bond-bending term, and $\sigma$ is the stretching-bending term. The units of $\alpha, \beta$, and $\sigma$ are $10^{3}$ dyne.

\begin{tabular}{lcccc}
\hline \hline \multicolumn{1}{c}{ System } & $d_{\text {bond }}^{0}(\AA)$ & $\alpha$ & $\beta$ & $\sigma$ \\
\hline For band-gap bowing & & & & \\
BAs & 2.0685 & 76.26 & 19.12 & -9.12 \\
GaAs & 2.4480 & 32.15 & 9.37 & -4.10 \\
For mixing enthalpy & & & & \\
BAs & 2.0685 & 76.26 & 22.2 & \\
GaAs & 2.4480 & 41.19 & 8.94 & \\
GaN & 1.9520 & 96.30 & 14.8 & \\
\hline \hline
\end{tabular}

TABLE V. Enthalpies of formation (LDA and VFF) of ordered (001) superlattices of $\mathrm{BAs} / \mathrm{GaAs}$ and the mixing enthalpies of

\begin{tabular}{|c|c|c|c|}
\hline System & $\begin{array}{c}\text { Boron } \\
\text { concentration }\end{array}$ & $\begin{array}{c}\Delta H \\
\text { (meV/atom) } \\
(\mathrm{LAPW})\end{array}$ & $\begin{array}{c}\Delta H \\
\text { (meV/atom) } \\
(\mathrm{VFF})\end{array}$ \\
\hline \multicolumn{4}{|c|}{ Ordered superlattices: } \\
\hline$(\mathrm{BAs})_{1} /(\mathrm{GaAs})_{1}(001)$ & $50 \%$ & 186 & 193 \\
\hline$(\mathrm{BAs})_{2} /(\mathrm{GaAs})_{2}(001)$ & $50 \%$ & 171 & 192 \\
\hline$(\mathrm{BAs})_{4} /(\mathrm{GaAs})_{4}(001)$ & $50 \%$ & 161 & 191 \\
\hline$(\mathrm{BAs})_{\infty} /(\mathrm{GaAs})_{\infty}(001)$ & $50 \%$ & 148 & \\
\hline$(\mathrm{BAs})_{1} /(\mathrm{GaAs})_{3}(001)$ & $25 \%$ & 132 & 174 \\
\hline \multicolumn{4}{|c|}{ Random alloys: } \\
\hline $\mathrm{BGa}_{7} \mathrm{As}_{8}(\mathrm{fcc})$ & $12.5 \%$ & 73 & 79 \\
\hline $\mathrm{BGa}_{15} \mathrm{As}_{16}(\mathrm{bcc})$ & $6.25 \%$ & 22 & 30 \\
\hline $\mathrm{BGa}_{31} \mathrm{As}_{32}$ (simple cubic) & $3.13 \%$ & 9.5 & 16 \\
\hline
\end{tabular}
$\mathrm{B}_{x} \mathrm{Ga}_{1-x}$ As random alloys [Eq. (9)]. All the $\Delta H$ values are positive.

$\left(\varepsilon_{s}^{B}-\varepsilon_{s}^{A s}\right)^{2}$, as described by Eq. (12). As is evident from Fig. 4, a small $\Gamma_{15 \mathrm{v}}-\Gamma_{15 \mathrm{c}}$ bonding/antibonding repulsion will lower the $\Gamma_{15 \mathrm{c}}$ state and a large $\Gamma_{1 \mathrm{v}}-\Gamma_{1 \mathrm{c}}$ bonding/ antibonding repulsion will raise the $\Gamma_{1 \mathrm{c}}$. In BAs these two effects are enough to reverse the ordering of the $\Gamma_{15 \mathrm{c}}$ and $\Gamma_{1 \mathrm{c}}$ states.

TABLE VI. Comparison of BAs band energies for LDA and GW (Ref. 24). Two LDA values are given: all-electron LAPW values from this paper and pseudopotential plane-wave (PP) values (Ref. 24). All the calculations used the experimental lattice constant of $4.777 \AA$. The exchange-correlation potential used in the PP calculations was not reported. For our LAPW calculations, the exchange-correlation of Perdew and Wang (Ref. 34) was used (see Sec. II for details.)

\begin{tabular}{lccc}
\hline \hline & LDA-PP $(\mathrm{eV})$ & LDA-LAPW $(\mathrm{eV})$ & $\mathrm{GW}(\mathrm{eV})$ \\
\hline$\Gamma_{6 c}$ & 4.5 & 4.57 & 5.5 \\
$\Gamma_{8 c}(\times 2)$ & 3.3 & 3.26 & 4.2 \\
$\Gamma_{7 c}$ & 3.1 & 3.05 & 4.0 \\
$\Gamma_{8 v}(\times 2)$ & 0.00 & 0.00 & 0.00 \\
$\Gamma_{7 v}$ & -0.22 & -0.21 & -0.22 \\
$\Gamma_{6 v}$ & -15.5 & -15.46 & -16.7 \\
$X_{6 c}$ & 11.4 & 11.27 & 13.1 \\
$X_{7 c}$ & 11.2 & 11.13 & 12.9 \\
$X_{6 c}$ & 1.38 & 1.274 & 1.86 \\
$X_{7 c}$ & 1.36 & 1.359 & 1.93 \\
$X_{6 v}$ & -4.1 & -4.07 & -4.5 \\
$X_{7 v}$ & -4.2 & -4.21 & -4.6 \\
$X_{7 v}$ & -8.6 & -8.49 & -9.5 \\
$X_{6 v}$ & -11.3 & -11.35 & -12.2 \\
$L_{6 c}$ & 8.8 & 8.65 & 9.8 \\
$L_{4,5 c}$ & 4.8 & 4.74 & 5.7 \\
$L_{6 c}$ & 4.7 & 4.66 & 5.6 \\
$L_{6 c}$ & 2.6 & 2.60 & 3.3 \\
$L_{4,5 v}$ & -1.8 & -1.81 & -2.0 \\
$L_{6 v}$ & -1.9 & -1.96 & -2.1 \\
$L_{6 v}$ & -8.8 & -8.82 & -9.7 \\
$L_{6 v}$ & -12.6 & -12.62 & -13.6 \\
\hline \hline
\end{tabular}




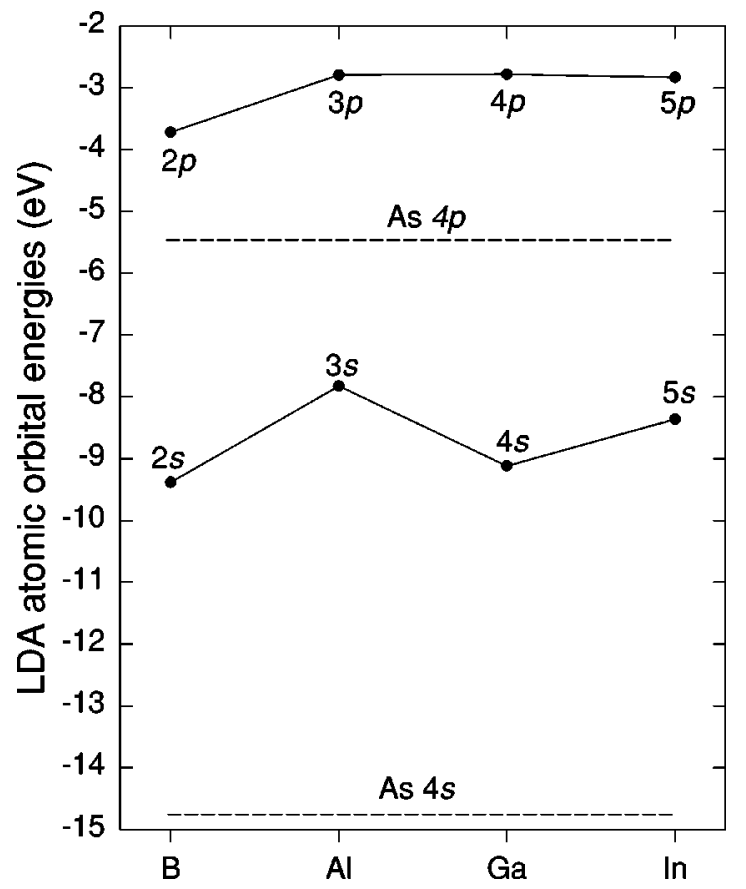

FIG. 1. Atomic $s$ and $p$ orbital energies in column III of the Periodic Table. Calculated fully relativistically within the localdensity approximation.

The large $\Gamma_{1 \mathrm{v}}-\Gamma_{1 \mathrm{c}}$ bonding/antibonding difference is due to the large $E_{s s}$ due to the short bond length in BAs. Empirical tight binding has also shown that the $\Gamma_{1 \mathrm{v}}-\Gamma_{1 \mathrm{c}}$ repulsion is much more pronounced in $\mathrm{BN}, \mathrm{BP}$, and $\mathrm{BAs}$ than in other Group-III-V's. ${ }^{23}$ The small $\Gamma_{15 \mathrm{v}}-\Gamma_{15 \mathrm{c}}$ bonding/antibonding repulsion is due to the small differences in the $p$ atomic orbitals of the boron and arsenic, as shown in Fig. 1. That is, the first term under the radical in Eq. (11) is small relative to other Group-III-V's.

The strong hybridization of the B and As $p$ states is visible in the partial densities-of-states plot [Eq. (2)] in Fig. 5

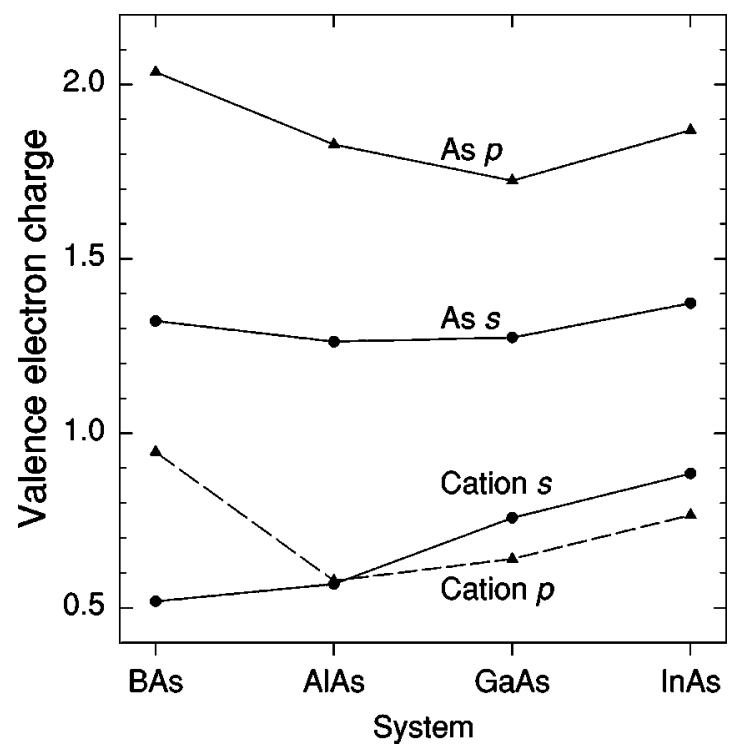

FIG. 2. Total valence charge [Eq. (4)] enclosed in spheres having the tetrahedral radii of Phillips (Ref. 37): boron $0.853 \AA$, aluminum $1.230 \AA$, gallium and arsenic $1.225 \AA$, and indium $1.405 \AA$.

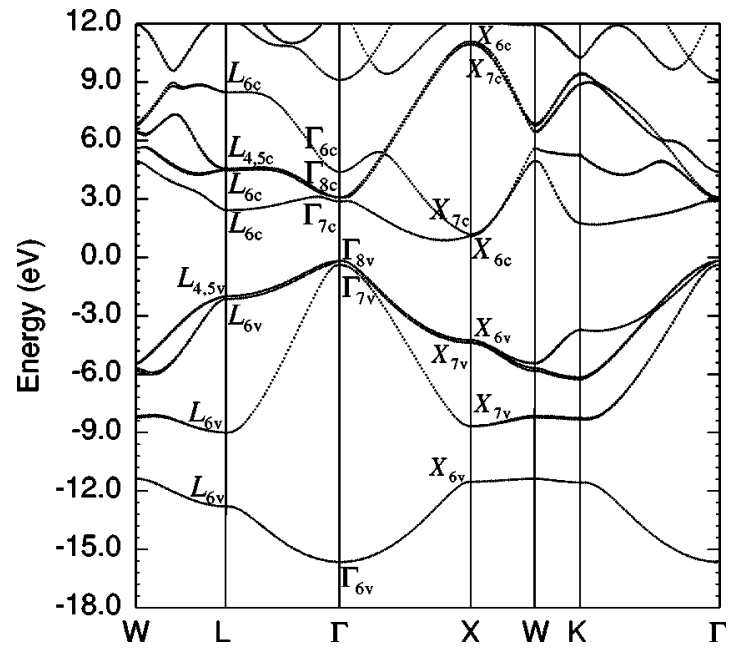

FIG. 3. Relativistic energy-band structure of BAs. The VBM is taken to be zero and the lattice constant is $4.777 \AA$.

and band character plots in Fig. 6 [Eq. (1)] where the width of the lines indicates the amount of the character indicated in the plot label. From these plots, one can see that the $s$ band of arsenic between -12 and $-16 \mathrm{eV}$ is distinct and has very little mixing with other states. This is similar to the other three Group-III-As systems, although in the case of BAs the arsenic $s$ band is wider and somewhat lower in energy. Two unique features of the BAs DOS compared to other Group-III-V semiconductors is the pronounced boron $p$ character at the VBM and anion $p$ character at the CBM. The stronger $p-p$ hybridization in BAs relative to the rest of the Group-III-As is due to the proximity of the orbital energies of B and As and the short bond length in the compound.

\section{Trends in band-gap and intervalley energy differences along the Group-III-As family}

Figure 7 shows the LDA-calculated vs experimental band gaps for the Group-III-As family, $M \operatorname{As}(M=\mathrm{B}, \mathrm{Al}, \mathrm{Ga}, \mathrm{In})$. For all but BAs, LDA energy band-gaps are taken from the LAPW calculations reported in Ref. 44. Experimental bandgaps are from Ref. 45. For BAs, for which no reliable experimental data exists, we used the $G W$ band-gap from Ref. 24 . Because of the unusual ordering of the conduction bands in BAs, the direct gap is not the $\Gamma_{15 \mathrm{v}} \rightarrow \Gamma_{1 \mathrm{c}}$ gap but the $\Gamma_{15 \mathrm{v}} \rightarrow \Gamma_{15 \mathrm{c}}$ gaps. The figures show the $\Gamma_{15 \mathrm{v}} \rightarrow \Gamma_{1 \mathrm{c}}$ gaps (which are the direct gaps in AlAs, GaAs, and InAs). We find in Fig. 7 that the LDA vs experimental $\Gamma_{15 \mathrm{v}} \rightarrow \Gamma_{1 \mathrm{c}}$ and the $\Gamma_{15 \mathrm{v}} \rightarrow L_{\mathrm{c}}$ gaps lie almost on a straight line. The LDA error of these gaps in the Group-III-As family is approximately constant, even for BAs. For the $\Gamma_{15 \mathrm{v}} \rightarrow \Gamma_{1 \mathrm{c}}$ gaps, the magnitude of the error is $\sim 1-1.5 \mathrm{eV}$. For the $\Gamma_{15 \mathrm{v}} \rightarrow L_{\mathrm{c}}$ gaps, the magnitude of the error is $\sim 0.7-1.1 \mathrm{eV}$. The experimental $\Gamma_{15 \mathrm{v}} \rightarrow X_{\mathrm{c}}$ gaps only vary by $\sim 0.5 \mathrm{eV}$ through the series and corresponding LDA gaps are nearly identical, resulting in a tight clustering of the $\Gamma_{15 \mathrm{v}} \rightarrow X_{\mathrm{c}}$ points in Fig. 7.

In Fig. 8(a), we show the $\Gamma_{15 \mathrm{v}} \rightarrow \Gamma_{1 \mathrm{c}}, \Gamma_{15 \mathrm{v}} \rightarrow L_{\mathrm{c}}$, and $\Gamma_{15 \mathrm{v}} \rightarrow X_{\mathrm{c}}$ gaps for the Group-III-As family. We see: (i) the ordering of the conduction-band states in BAs and AlAs is $X_{1 \mathrm{c}}<L_{1 \mathrm{c}}<\Gamma_{1 \mathrm{c}}$ whereas (ii) the ordering is $\Gamma_{1 \mathrm{c}}<L_{1 \mathrm{c}}<X_{1 \mathrm{c}}$ 


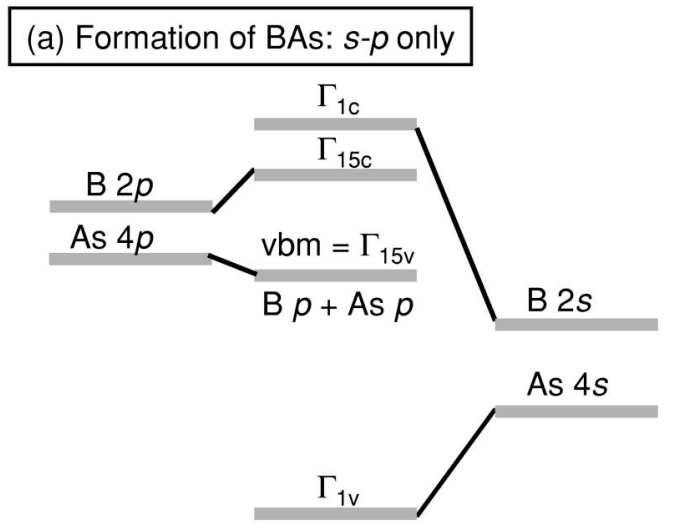

(b) AlAs - GaAs - InAs: $s p+d$

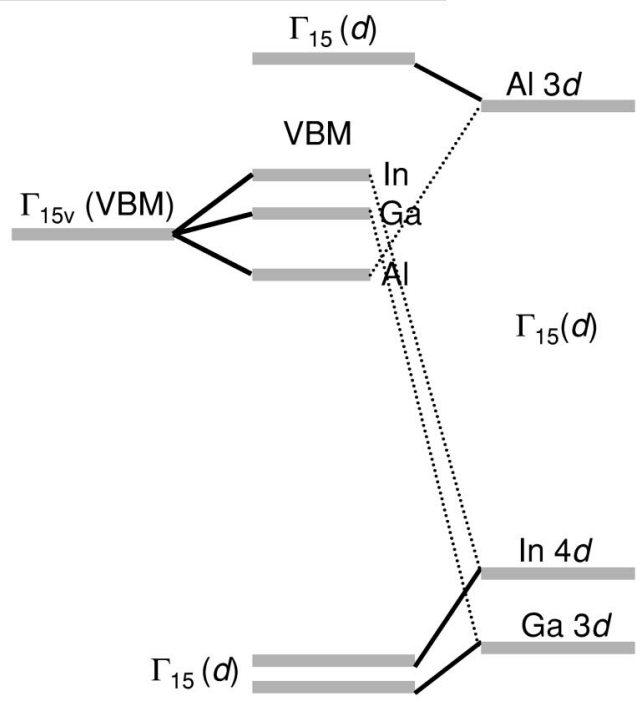

FIG. 4. Schematic diagram of energy levels in the Group-III-As series. (a) Schematic diagram of energy levels in zincblende BAs and their atomic origins. Because the B- $p-\mathrm{As}-p$ orbital energy difference is small relative to other Group-III-V compounds and the B-s-As-s orbital energy difference is large, the $p$-like $\Gamma_{15 \mathrm{c}}$ conduction-band state is below the $s$-like $\Gamma_{1 \mathrm{c}}$ conduction-band state. This ordering of the lower conduction-band states is unusual for Group-III-V compounds and is reminiscent of silicon. (b) Schematic diagram of the effects of $p-d$ coupling on the VBM in AlAs, GaAs, and InAs. The VBM is driven up by the coupling of the $3 d(4 d)$ states in GaAs(InAs) and the As $4 p$-like VBM, but in AlAs, this $p$ - $d$ repulsion drives the VBM down.

for GaAs and InAs, (iii) the $X_{1 \mathrm{c}}$ and $L_{1 \mathrm{c}}$ states are closely spaced in AlAs and GaAs, (iv) BAs is "strongly indirect"; $L_{1 \mathrm{c}}$ and $\Gamma_{1 \mathrm{c}}$ are far above the CBM (which is close to $X_{1 \mathrm{c}}$ ), and (v) a crossing of the lowest $X$ and $L$ conduction states occurs between AlAs and GaAs, but the $\Gamma_{15 \mathrm{v}} \rightarrow \Gamma_{1 \mathrm{c}}$ gaps decrease so rapidly in the series that the $\Gamma_{1 \mathrm{c}}$ states lie below the $L_{1 \mathrm{c}}$ in GaAs and InAs, and thus these two members of the family are direct band-gap materials (whereas BAs and AlAs are indirect $X$ gap materials).

Figure 8(b) shows the conduction-band intervalley differences $\Gamma_{1 \mathrm{c}} \rightarrow X_{\mathrm{c}}$ and $\Gamma_{1 \mathrm{c}} \rightarrow L_{1 \mathrm{c}}$. Both differences show a smoothly decreasing trend from AlAs to GaAs to InAs, but an anomalously large decrease from BAs to AlAs. Again, this is a result of the enhanced $s-s$ repulsion in BAs. Be-

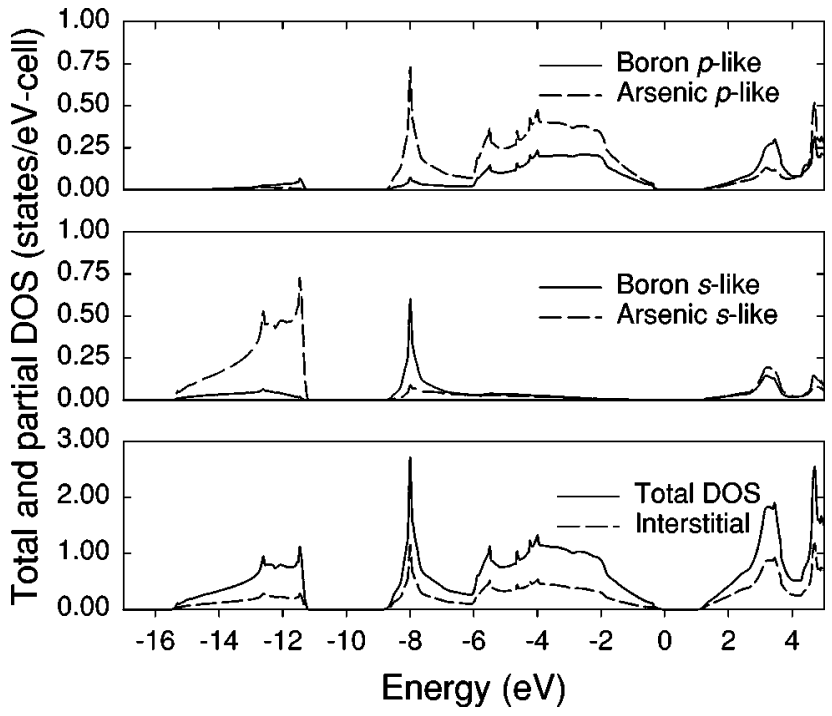

FIG. 5. Partial and total densities-of-states for BAs [Eq. (2)] inside muffin-tin spheres with radii $0.853 \AA$ for boron and $1.225 \AA$ for arsenic (Ref. 37). Note the strong mixing of B and As $p$ states. Figure 2 shows a quantitative measure of the $p-p$ mixing in each of the Group-III-As compounds.

cause the $\Gamma_{1 \mathrm{c}}$ state has much more $s$ character than either the lowest $X$ and $L$ conduction states (see Fig. 6), the effect of the $s-s$ repulsion is greater for the $\Gamma_{1 \mathrm{c}}$ than for these other two states, driving it up relative to them. This effect is more pronounced for the $\Gamma_{1 \mathrm{c}} \rightarrow X_{\mathrm{c}}$ difference because the lowest $X$ conduction state has less $s$ character than the lowest $L$ conduction state.

\section{Charge density and ionicity trends in the Group-III-As family}

The valence charge density [upper four bands, $d$ bands not included; Eq. (4)] in the (110) plane is shown for the four members of the Group-III-As family in Fig. 9. We see that the charge densities of AlAs, GaAs, and InAs are drawn towards the anion and exhibit a "single hump" in the bond charge. (The relative shifts in the bond charge, $d_{\max } / d_{\text {bond }}$, are $\sim 0.68$ for AlAs and GaAs, and $\sim 0.71$ for InAs.) The relative shift of charge towards the anion (As) is due to the difference in electronegativity of the anion and the cation and reflects the partially ionic nature of the bond. In contrast, because of the almost entirely covalent nature of the bonding in the BAs system, in this system the charge density features a "double hump" bond similar to the bond charges in diamond $\mathrm{C}$ and $\mathrm{Si}^{46,47} \mathrm{~A}$ similar analysis of the charge density of BAs was reported earlier by Wentzcovitch and Cohen. ${ }^{20}$ We see that BAs is unique in the Group-III-As family in that the bonding is very covalent. This is evidenced by the exceptionally strong hybridization of $p$ states from the anion and cation (see Figs. 2, 5, and 6) and the significant bonding charge visible in the valence charge density, which is similar to the bonding charge in diamond $\mathrm{C}$ and $\mathrm{Si}$.

It is interesting to note that, in contrast to $\mathrm{AlAs}, \mathrm{GaAs}$, and InAs where charge is drawn towards the anion, the nearly symmetric bond charge distribution shown in the valence charge plot of BAs is not centered exactly around the bond center but is actually drawn slightly toward the cation 

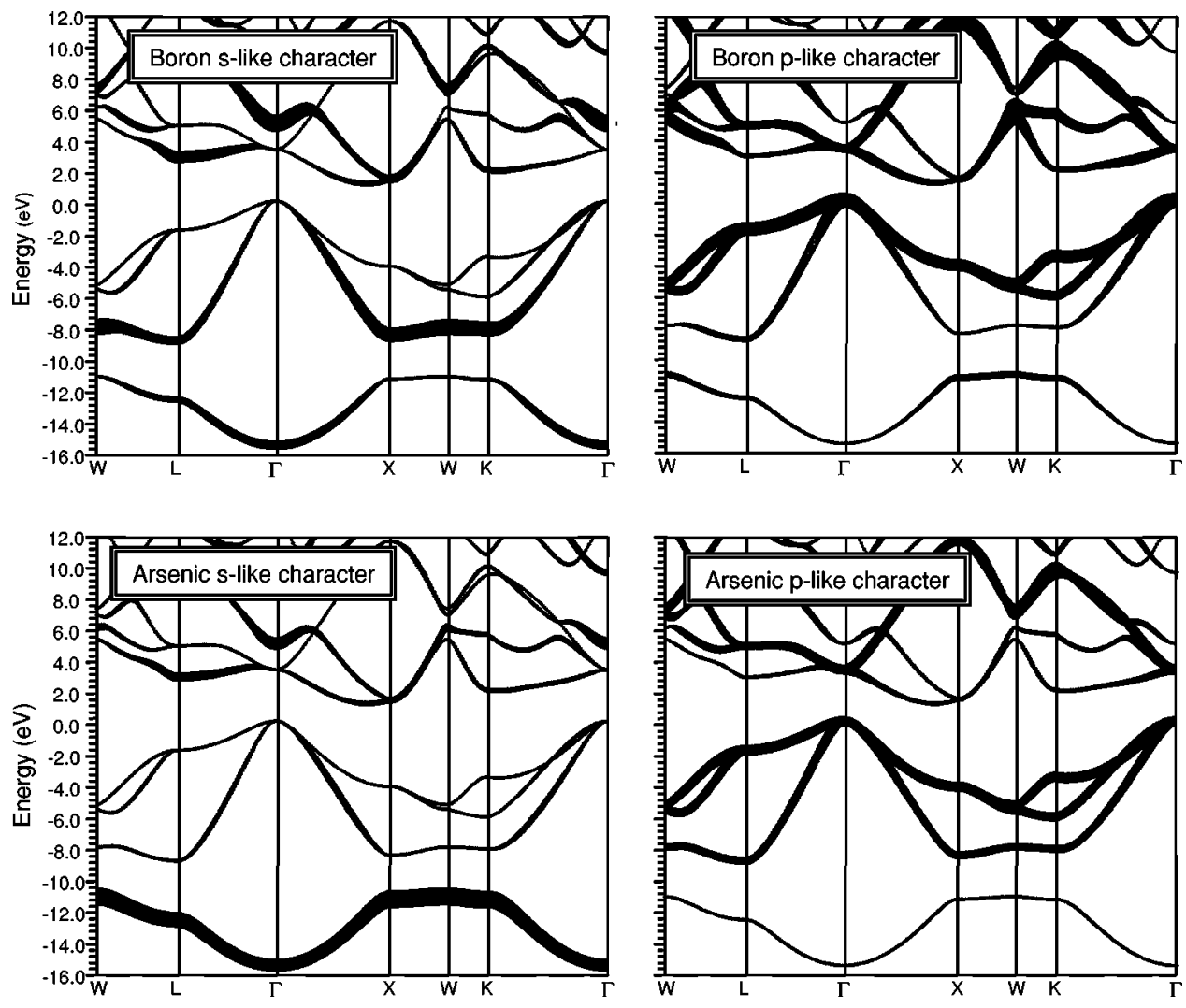

FIG. 6. Band characters of BAs [Eq. (1)] plotted as a function of $k$. The thickness of the lines denotes a relative amount of a given character. The $\mathrm{B}$ and As $p$ states are stongly mixed at both the VBM and the CBM whereas the As $s$ band is distinct. There is some mixing of As $p$ and B $s$ states in the bottom of the $p-p$ hybridized bands.
B. (The distance between the bond center and the boron position is about $45 \%$ of the total bond length.) This would indicate that whatever small ionic component exists in BAs results not from charge transfer towards the anion but from charge transfer towards the cation B. Further evidence of an ionic component in BAs where the boron atom acts as the anion is shown in the valence charge difference plots [Eq. (5)] in Fig. 10. In AlAs, GaAs, and InAs, the maximum (marked as a cross) in $\Delta \rho_{\text {val }}$ occurs closer to the As anion than to the cation, consistent with the reported ionicities. However, in BAs, the maximum in $\Delta \rho_{\text {val }}$ occurs closer to the $\mathrm{B}$ atom. In addition, the positive region around the $\mathrm{B}$ atom in $\Delta \rho_{\text {val }}$, which does not occur for the other members of the Group-III-As family, is further indication of a slight ionic component in BAs where B plays the role of the anion rather than the cation. This reversal of the cation and anion roles in BAs (and in BP) is predicted by the Phillips scale of electronegativities $^{42}$ and has been discussed previously by Wentzcovitch and Cohen. ${ }^{20}$

\section{BAND OFFSETS: BAs/GaAs/AIAs}

The offset $\Delta E_{v}(A X / B Y)$ between the valence-band maxima of two semiconductor compounds $A X$ and $B Y$ forming a heterostructure is one of the most important parameters in interfacial structures deciding both transport and quantum confinement. The results for the natural (unstrained) band offsets [Eq. (6)] for BAs/GaAs and BAs/AlAs are plotted in Fig. 11. The conduction-band offsets shown in the figure are determined by adding the experimental gaps to the calculated valence-band offsets. The natural band offset for AlAs/GaAs has been computed previously ${ }^{48}$ and has been included here to contrast the offset behavior of "typical" heterojunctions and those of heterojunctions with BAs.

The ordering of the VBM of BAs in the Group-III-As is somewhat surprising. Because the VBM decreases systematically in the order InAs $\rightarrow \mathrm{GaAs} \rightarrow \mathrm{AlAs}$ [Fig. 11(b)], one might expect that the VBM of BAs would lie below AlAs, but in fact, the VBM of BAs is above AlAs, nearly as high as GaAs. The ordering of the VBMs for InAs, GaAs, and AlAs can be understood qualitatively using a tight-binding argument analogous to Eq. (11) for $\Gamma_{15 \mathrm{c}}$ when a $p$ - $d$ term is added. The effects of this additional term are indicated schematically in Fig. 4(b). The ordering of the VBM's is as follows:

InAs relative to GaAs: Two effects account for the slightly higher VBM of InAs relative to that of GaAs. ${ }^{49}$ (i) InAs has a longer bond length than GaAs. This reduces $E_{p p}$ of Eq. (11) and tends to drive the $\Gamma_{15 \mathrm{v}}$ VBM up relative to GaAs. (ii) In tetrahedral symmetry, the cation $d$ and anion $p$ states share the same $\Gamma_{15}$ symmetry and hence can interact through the potential matrix element $\left\langle\phi_{\text {cation }}^{d}|V| \phi_{\text {anion }}^{p}\right\rangle$ $=E_{d p}$. The mostly anion $p$-like VBM is repelled upwards by the $\mathrm{Ga} / \mathrm{In} d$ states by an amount $E_{d p}^{2} /\left(\varepsilon_{p}^{\text {anion }}-\varepsilon_{d}^{\text {cation }}\right)$. This $p-d$ repulsion ${ }^{49,48}$ is slightly stronger in InAs where the cation $4 d$ states are shallower and less localized than the Ga $3 d$ state, as shown schematically in Fig. 4(b).

GaAs relative to AlAs: The difference between the VBM of GaAs and AlAs can also be understood in terms of this $p-d$ repulsion effect. In GaAs, the deep gallium $d$ states couple to the arsenic $p$ states, driving the VBM up, but in AlAs, there are no aluminum $d$ states below the VBM. Instead, the interaction of the arsenic $p$ states at the VBM and the unoccupied aluminum $d$ states above the VBM drives the AlAs VBM down. Thus, $p$ - $d$ repulsion has the opposite effect on the VBM in GaAs and AlAs [Fig. 4(b)]. 


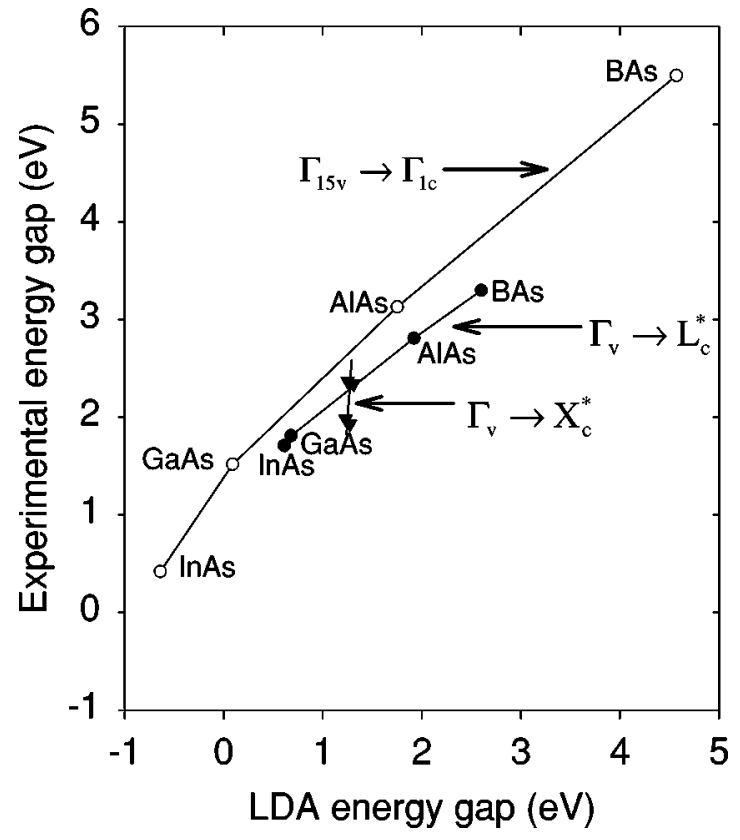

FIG. 7. Trends in the LDA vs experimental band-gaps of the Group-III-As family. For the $\Gamma_{15 \mathrm{v}} \rightarrow \Gamma_{1 \mathrm{c}}$ and the $\Gamma_{15 \mathrm{v}} \rightarrow L_{\mathrm{c}}$ gaps, the errors are nearly constant. The LDA errors are $\sim 1-1.5 \mathrm{eV}$ and $\sim 0.7-1.1 \mathrm{eV}$ for the $\Gamma_{15 \mathrm{v}} \rightarrow \Gamma_{1 \mathrm{c}}$ and the $\Gamma_{15 \mathrm{v}} \rightarrow L_{\mathrm{c}}$ gaps, respectively.

AlAs relative to BAs: Two effects increase the $\mathrm{VBM}$ of BAs relative to the VBM of AlAs. (i) Because the unoccupied $d$ states in BAs lie very high in energy relative to the VBM, the $p$ - $d$ repulsion effect that drives the VBM down in AlAs is weaker in BAs. (ii) The more important effect is the unusual character of the VBM in BAs. In the rest of the Group-III-As family, the character of the VBM is primarily anion $p$-like, but in BAs the bonding is much more covalent and the VBM comes from both the anion and the cation (Fig. $6)$. Since the cation $p$ levels are higher in energy than the As $p$ levels (Fig. 1), any admixture of cation $p$ character pulls the VBM up. These two effects, lack of $p$ - $d$ repulsion and strong hybridization of the cation $p$ character into the VBM, account for the high VBM of BAs relative to AlAs.

GaAs relative to BAs: While the VBM of BAs and GaAs both lie above that of AlAs for the reasons given above, the VBM of BAs lies only slightly below that of GaAs despite the much smaller lattice constant $(\sim 17 \%$ mismatch $)$ and the lack of $p-d$ repulsion. This is due to the unusual cation $p$ hybridization into the VBM of BAs, which raises the VBM by several tenths of an eV.

Finally, we note that the transitivity among the band offsets of BAs/AlAs/GaAs is similar to other compounds where the lattice mismatches are not as large. By transitivity we mean that the band offset $\Delta E_{v}(A / C)$ between two compounds $A$ and $C$ is well approximated as the sum of the band offsets between compounds $A$ and $B$ and between compounds $B$ and $C$, i.e.,

$$
\begin{aligned}
\Delta E_{v}(\mathrm{GaAs} / \mathrm{BAs}) \cong & \Delta E_{v}(\mathrm{BAs} / \mathrm{AlAs}) \\
& +\Delta E_{v}(\mathrm{AlAs} / \mathrm{GaAs})
\end{aligned}
$$

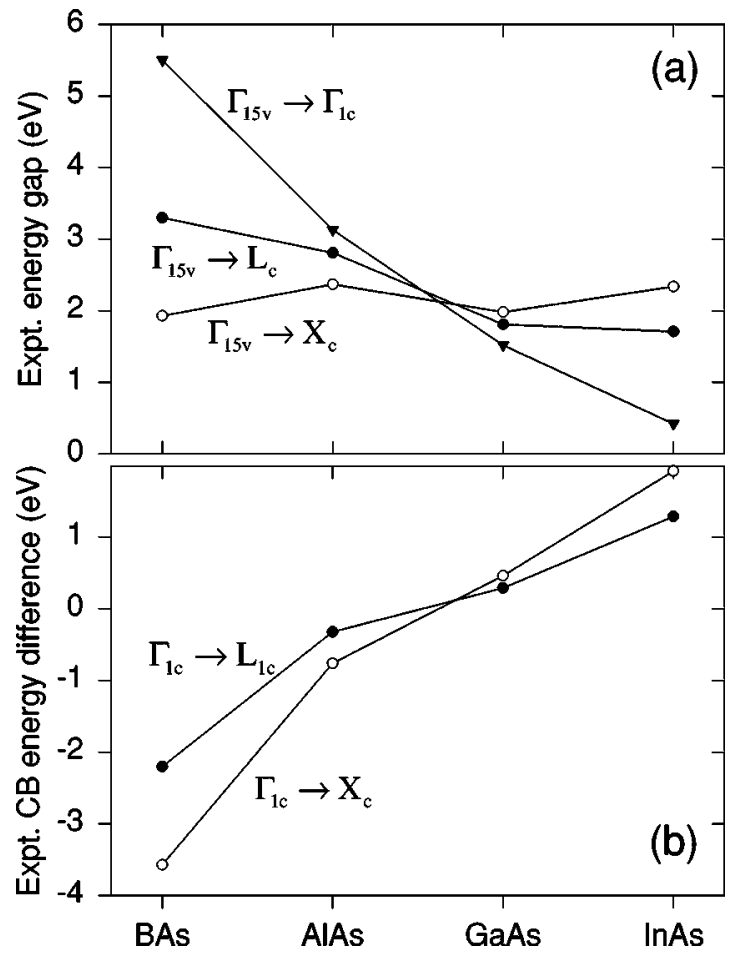

FIG. 8. Trends in the intervalley energy seperations of the Group-III-As family. Note the crossing of the $\Gamma_{15 \mathrm{v}} \rightarrow X_{\mathrm{c}}$ gaps and the $\Gamma_{15 \mathrm{v}} \rightarrow \Gamma_{1 \mathrm{c}}$ gaps between AlAs and GaAs. Thus, GaAs and InAs are direct gap materials whereas BAs and AlAs are indirect $X$ gap materials.

In this case, $\Delta E_{v}(\mathrm{GaAs} / \mathrm{BAs})$ calculated directly, yields 0.19 $\mathrm{eV}$ but $\Delta E_{v}(\mathrm{BAs} / \mathrm{AlAs})+\Delta E_{v}(\mathrm{AlAs} / \mathrm{GaAs})$ yields $0.12 \mathrm{eV}$, a nontransitivity difference of $0.07 \mathrm{eV}$.

\section{BAs-GaAs ALLOYS}

\section{A. Bond lengths and bond angles in the alloys}

Interest in BAs-GaAs alloys centers around the hope that boron will modify the GaAs band-gap similarly to nitrogen without the adverse effect ${ }^{50}$ of introducing localized states that reduce carrier diffusion length. We know from the theory of Group-III-V alloys ${ }^{29,51}$ that the optical properties are decided by both the atomic relaxation and by charge transfer. Thus, we first study the bond relaxation around a boron substitutional impurity.

The bond lengths and angles in the (110) plane of GaAs with $\sim 3 \%$ boron substitution $\left(\mathrm{B}_{1} \mathrm{Ga}_{31} \mathrm{As}_{32}\right.$ supercell) are shown in Fig. 12. Bond lengths are shown as a percentage of the pure bulk GaAs bond length, except for the B-As bonds where the values indicate a percentage of the pure bulk BAs bond length. The numbers that lie between a triplet of atoms indicate the bond angle between the three atoms as a percentage of the ideal bond angle in the zincblende structure of $109.47^{\circ}$. The rectangle represents the supercell boundary. We note several observations:

(i) Bond angles near boron increase by up to $5 \%$ from their ideal value. This helps accommodate the Ga-As and B-As alloy bonds (hereafter $R_{\mathrm{GaAs}}$ and $R_{\mathrm{BAs}}$, respectively) to keep the bond lengths close to their ideal values, $R_{\mathrm{BAs}}^{0}$ and $R_{\mathrm{GaAs}}^{0}$ in the pure binary compounds. 

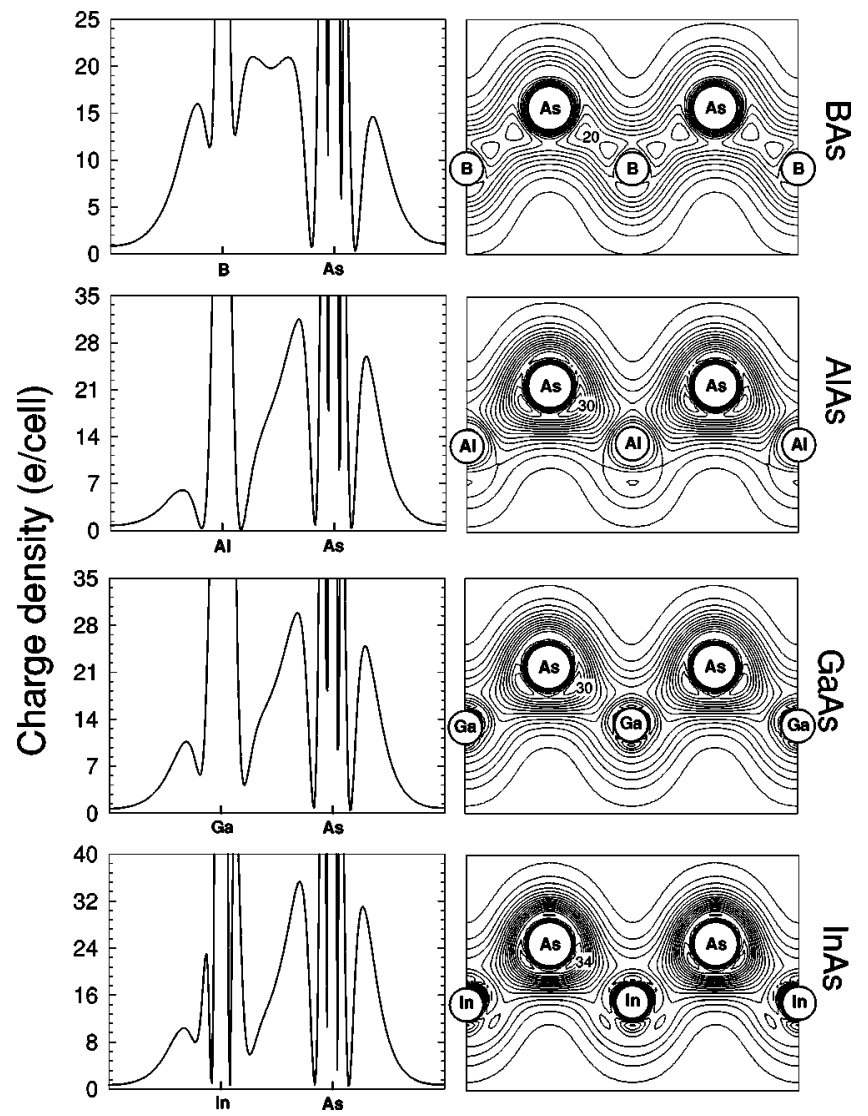

FIG. 9. Valence charge densities $\rho_{\text {val }}$ [Eq. (4)] for the GroupIII-As family, excluding the cation and anion $d$ states. The contour plots use the same scale as the corresponding line plots. The contour spacing is 2 e/cell. The range of the line plots is from one tetrahedral interstitial site, through the bond, and to the neighboring tetrahedral interstitial site. Note the striking asymmetry of the bonding charge for the three systems AlAs, GaAs, and InAs, while the bond charge in BAs reveals a covalent, double-hump feature similar to that found in diamond $\mathrm{C}, \mathrm{Si}$, or $\mathrm{Ge}$.

(ii) The B-As bond decreases from the GaAs value towards the BAs value and ends up only $4 \%$ higher than the value for pure BAs.

(iii) The average bond length relaxation parameter ${ }^{52}$

$$
\epsilon=\left[R_{\mathrm{GaAs}}(x)-R_{\mathrm{BAs}}(x)\right] /\left[R_{\mathrm{GaAs}}^{0}-R_{\mathrm{BAs}}^{0}\right]
$$

is 0.76. ( $\epsilon=0$ when there is no relaxation and 1 when the relaxation is full.) Thus, assuming $R_{\mathrm{BAs}}$ to be Vegard-like (as in the virtual crystal approximation) overestimates the bond length by $\sim 13 \%$.

We also modeled a $50 \%-50 \%$ random alloy using a $32-$ atom special quasirandom structure (SQS16 in Table II). ${ }^{39}$ We found that the distribution of the bond lengths has the expected bimodal form for a random binary alloy, ${ }^{53}$ and the B-As bonds are generally larger than the ideal B-As bond length while the Ga-As bond lengths are smaller than the ideal Ga-As bond length.

\section{B. Bowing in the dilute alloy}

Both measurements and calculations indicate that, for isovalent semiconductor alloys, the deviation of the band gap
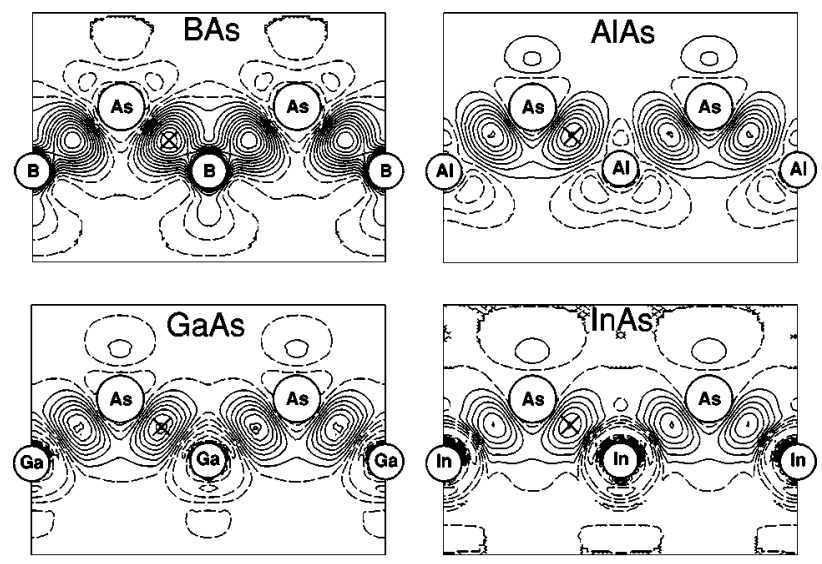

FIG. 10. Deformation density $\Delta \rho_{\text {val }}\left(\rho_{\text {val }}^{\text {solid }}-\rho_{\text {val }}^{\text {atom }}\right)$ plots for the Group-III-As family in the (110) plane. Contours marked with dashed lines correspond to negative values of $\Delta \rho_{\mathrm{val}}$. The units are $\mathrm{e} / \AA^{3}$ and the contour spacing is 0.02 . The maxima are marked by crosses. To determine $\Delta \rho_{\text {val }}$, the overlapping charge densities of free atoms are subtracted from the self-consistent charge density of the solid. The atomic configurations for the free atoms are $d^{10} s^{2} p^{1}$ and $s^{2} p^{3}$ for the cations and anions, respectively. The $d$ bands of the cations, but not of the anions, are included in the valence bands.

$\Delta E_{g}(x)$, from the average band gap $\bar{E}_{g}(x)$, of the constituents is often well described by a quadratic term, ${ }^{29,51,54,55}$

$$
\Delta E_{g}(x)=b x(x-1)
$$

For typical semiconductor alloys, the bowing parameter $b$ is normally less than $1 \mathrm{eV}$ and is independent ${ }^{45}$ of the concentration $x$. However, in $\mathrm{GaAs}_{1-x} \mathrm{~N}_{x}$ alloys, where the lattice mismatch is large and the bond strength differences are significant, the bowing parameter is strongly composition dependent ${ }^{56}$ and can be as large as $20 \mathrm{eV}$. Due to the large lattice mismatch of BAs and GaAs and the strong B-As bonds, one might expect that $\mathrm{B}_{x} \mathrm{Ga}_{1-x} \mathrm{As}$ alloys would also show such large and composition-dependent bowing. We calculated the $\Gamma_{15 \mathrm{v}} \rightarrow \Gamma_{1 \mathrm{c}}$ gaps in the experimentally relevant composition range of $0 \%-10 \%$. The bowing was calculated using 64-atom supercells, as described in Sec. II. In this composition range, the direct $\Gamma_{15 \mathrm{v}} \rightarrow \Gamma_{1 \mathrm{c}}$ gap is the smallest gap. The bowing was calculated using the LDA $\Gamma_{15 \mathrm{v}} \rightarrow \Gamma_{1 \mathrm{c}}$ gaps of BAs, GaAs (4.64 and $0.28 \mathrm{eV}$, respectively) and the LDA gaps at two alloy compositions, $3 \%$ and $6 \%$. At the $3 \%$ composition, the bowing is $b=3.4 \mathrm{eV}$ and at $6 \%$ the bowing is $b=3.6 \mathrm{eV}$. Thus, the band-gap bowing is $\sim 3.5 \mathrm{eV}$ and relatively composition independent in this composition range. A recent experimental study, ${ }^{18}$ estimated the bowing to be $b=1.6 \pm 0.3$ or $2.3 \pm 0.3$ using theoretical estimates for the direct gap of cubic BAs of 3.56 (Ref. 19) and $4.23 .^{22}$ However, because of the LDA error in the band gaps (see Fig. 7), these estimated band-gaps are too small, and thus, the bowing is underestimated. Because the LDA errors for the band-gaps are approximately constant for the GroupIII-As series, the bowing can be reliably calculated if all the points $\left(E_{A}, E_{B}\right.$, and $\left.E_{A_{1-x} B_{x}}\right)$ are taken consistently from LDA calculations (as we did) because only the relative values are important. For computing the band gap bowing from 


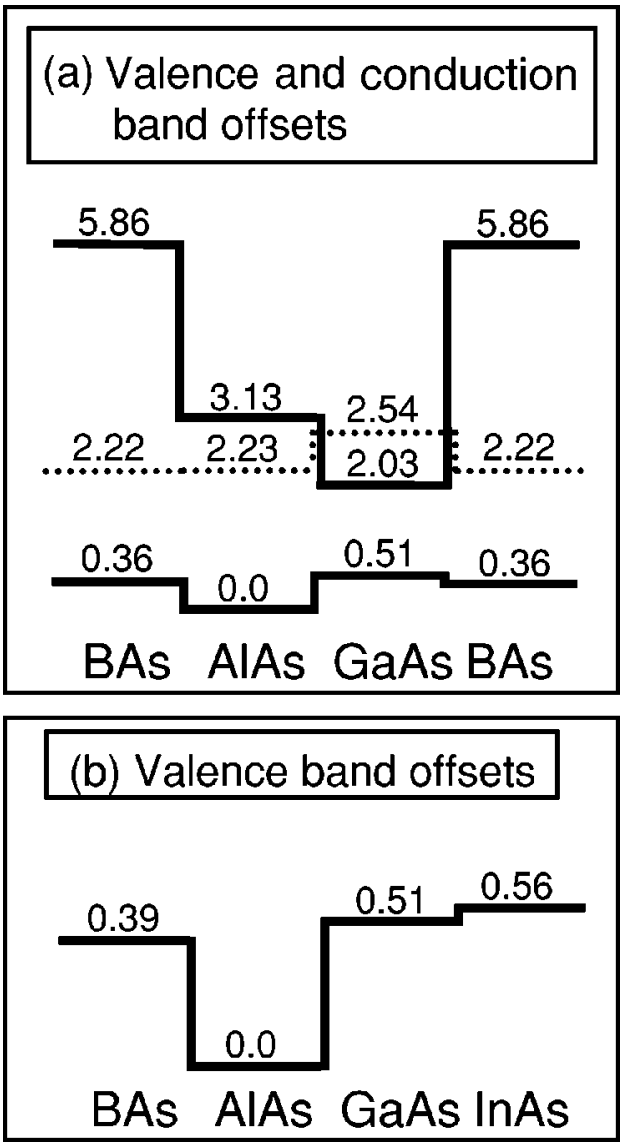

FIG. 11. Valence- and conduction-band offsets (in eV) for BAs/ GaAs/AlAs and for the Group-III-As family. The valence-band offsets are calculated directly using Eq. (6). The conduction-band offsets are obtained by adding the experimental band-gap values to the valence-band offsets. Part (a) shows both the valence-band and conduction-band offsets for BAs/AlAs, AlAs/GaAs, and GaAs/ BAs. Both the direct $\Gamma_{15 \mathrm{c}}$ gap (solid line) and the $X_{1 \mathrm{c}}$ gap (dotted line) are shown. The height of the BAs VBM was determined by averaging the valence-band offsets of BAs with respect to AlAs and GaAs. Part (b) shows the valence-band offsets in the Group-III-As family. Surprisingly, the VBM of BAs is found to be above that of AlAs and not very far below that of GaAs.

experimental alloy data, the $G W$ estimate of the BAs direct band gap $(5.5 \mathrm{eV}),{ }^{24}$ which corrects LDA errors, is a more appropriate value to use to calculate the bowing. Using the experimental alloy data from Ref. 18, the $G W$-estimated band-gap for BAs $(5.5 \mathrm{eV})$, and the experimental value of $1.42 \mathrm{eV}$ for GaAs, Geisz estimates ${ }^{57}$ the revised bowing parameter to be $\sim 3.5 \mathrm{eV}$ in excellent agreement with the theoretical prediction.

The bowing of $\mathrm{B}_{x} \mathrm{Ga}_{1-x}$ As is much smaller than the bowing for $\mathrm{GaAs}_{1-x} \mathrm{~N}_{x}$ alloys in the same range. Accordingly, we predict that addition of boron to GaAs will raise the gap. For example, the predicted gaps for $2 \%, 3 \%, 5 \%$, or $10 \%$ concentrations of boron, the band-gaps would be $1.43,1.44$, 1.46 , and $1.51 \mathrm{eV}$, respectively. Thus, the addition of boron to GaAs will not lead to a $1-\mathrm{eV}$-gap alloy as seen with nitrogen addition. We also find that the bowing is composition independent. This implies that the effects of boron on the band-gap in GaAs are much less pronounced than those of

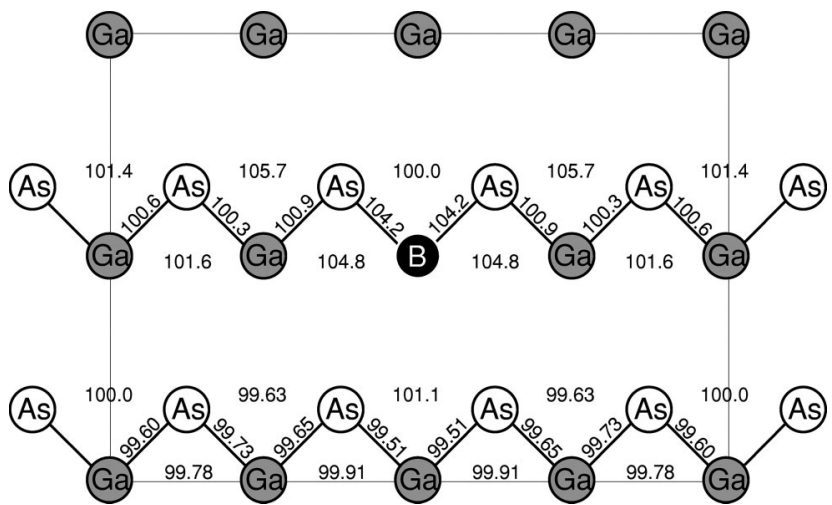

FIG. 12. Bond lengths and angles in $\mathrm{BGa}_{31} \mathrm{As}_{32}$ in the (110) plane. Bond lengths are shown as a percentage of the ideal GaAs bond length, except for the B-As bonds where the values indicate a percentage of the ideal BAs bond length. The numbers that lie between a triplet of atoms indicate the bond angle between the three atoms as a percentage of the ideal bond angle in the zincblende structure of $109.47^{\circ}$. The rectangle represents the supercell boundary.

nitrogen. This is not completely unexpected, however, as it has been previously observed that the bowing is smaller in mixed cation systems than in mixed anion systems. ${ }^{29}$

We have also calculated the band-gap of the $\mathrm{BGaAs}_{2}$ $\mathrm{CuPt}$-ordered compound finding that it is $0.12 \mathrm{eV}$ below the $50 \%-50 \%$ random alloy modeled via the SQS16.

\section{Enthalpy of mixing and stability of superlattices}

Table $\mathrm{V}$ gives the formation enthalpies [Eq. (9)] for (001) ordered superlattices of $\mathrm{BAs} / \mathrm{GaAs}$ and the mixing enthalpies of $\mathrm{B}_{x} \mathrm{Ga}_{1-x} \mathrm{As}$ random alloys. The formation energy of ordered superlattices of orientation $\hat{G}$ and period $n$ is

$$
\Delta H(n, \hat{G})=E\left(A_{n} B_{n} ; \hat{G}\right)-\frac{1}{2}\left(E_{A}+E_{B}\right)
$$

and can be seperated into two contributions: ${ }^{58}$ (i) The consituent strain energy, i.e., the energy of the infinite period superlattice. This is the strain energy associated with deform-

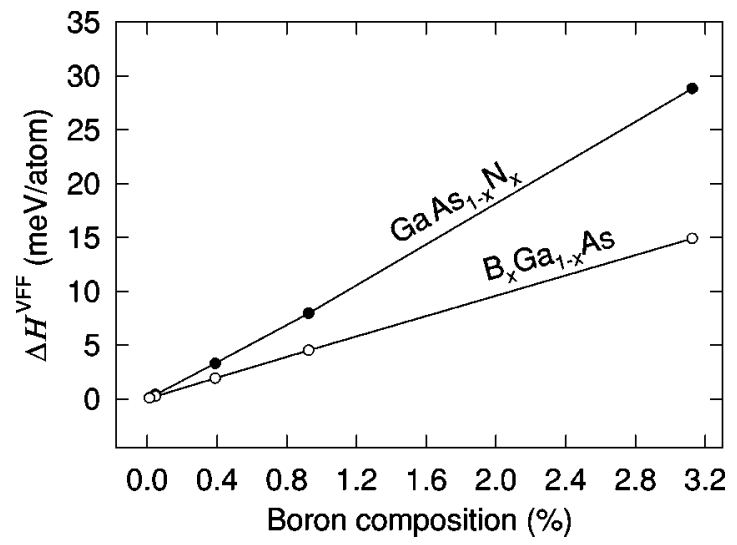

FIG. 13. Calculated VFF mixing enthalpies for dilute $\mathrm{GaAs}_{1-x} \mathrm{~N}_{x}$ alloys and dilute $\mathrm{B}_{x} \mathrm{Ga}_{1-x}$ As alloys. The mixing enthalpy is substantially smaller for boron alloys. 


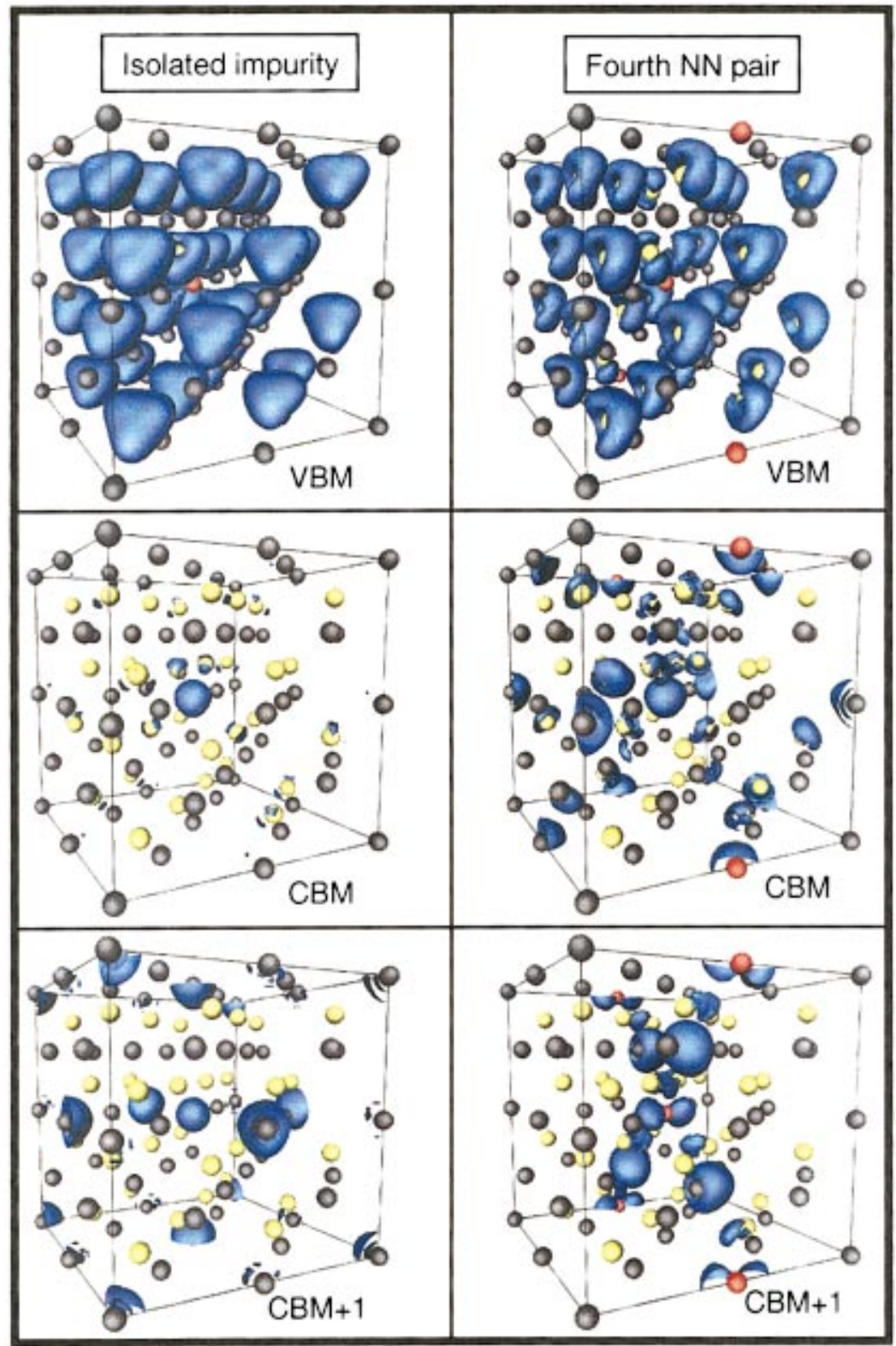

FIG. 14. (Color) Isosurfaces of the wave function squared for a single impurity and for a fourth nearest-neighbor pair. Boron atoms are red, gallium black, and arsenic yellow. The isosurface value (blue) is $0.01 \mathrm{e} / \AA^{3}$. In both cases, the VBM states are completely delocalized and are primarily As derived. The lower conductionband states are strongly concentrated around the boron atoms.

ing pure $A$ and pure $B$ into the in-plane lattice constant $\bar{a}$ of the superlattice, and relaxing them in the direction $\hat{G}$. (ii) The interfacial energy, i.e., the difference between $\Delta H(n, \hat{G})$ and the consituent strain energy, is defined by

$$
\Delta H(n, \hat{G})=\frac{2 I(n, \hat{G})}{n}+\Delta E_{C S}(\bar{a}, \hat{G}) .
$$

We calculated $\Delta E_{C S}(\bar{a}, \hat{G})$ for BAs and GaAs, deformed to the average lattice constant $\bar{a}$ along $\hat{G}=(001)$. This gave $148 \mathrm{meV} /$ atom. From $\Delta H(n, \hat{G}=001)$ of Table $\mathrm{V}$ and $\Delta E_{C S}$ we calculated the interfacial energy $I$ for $n=1,2$, and 4 . We found $I=19,23$, and $26 \mathrm{meV}$, respectively. While a larger superlattice period $n$ would be required to determine the converged value of $I$, we see that the BAs-GaAs interface is repulsive. This is why $\Delta H(n, \hat{G})$ of Table $\mathrm{V}$ decreases with $n$ : larger $n$ reduces the proportional effect of the interfacial repulsion.
So far we dealt with (001) superlattices. The CuPt-like monolayer (BAs) $)_{1} /(\mathrm{GaAs})_{1}(111)$ is particularly interesting since in other Group-III-V alloys (e.g., GaInP 2 ) it appears as a spontaneously ordered stucture during alloying. ${ }^{59} \mathrm{We}$ find a very high $\Delta H(\mathrm{CuPt})$ of $108 \mathrm{eV} /$ atom, suggesting thermodynamic instability.

For the random alloys in Table V, all of the excess enthalpies are positive. We compare the mixing enthaplies as obtained by the LDA (which includes both size-mismatchedinduced strain effects and charge-transfer "chemical', effects) and the valence force field (VFF) method (which includes only strain effects). The trends and magnitudes are similar, showing that the strain effects dominate $\Delta H$. In Fig. 13 we compare the VFF-calculated mixing enthalpy of nitrogen in GaAs and contrast it with that of boron in GaAs, showing that the mixing enthalpy of $\mathrm{GaAs}_{1-x} \mathrm{~N}_{x}$ alloys is much higher than for $\mathrm{B}_{x} \mathrm{Ga}_{1-x}$ As alloys. These results indicate that it may be easier to alloy boron with GaAs. This implies that the range of bulk $\mathrm{B}_{x} \mathrm{Ga}_{1-x}$ As alloy compositions could be substantially larger than for the nitride alloy 
$\mathrm{GaAs}_{1-x} \mathrm{~N}_{x}$. In epitaxial growth experiments, the alloy solubility can dramatically exceed that in bulk experiments for reasons explained in Ref. 60.

\section{Quasilocalized electronic states}

We find that the incorporation of boron into the GaAs host material has little effect on the state at the VBM but that the lower conduction-band states are strongly peturbed. The square of the wave functions for the VBM and the lowest two conduction-band states for isolated boron in GaAs are shown in the left-hand side of Fig. 14. The first column shows the states for an isolated boron atom in the center of a supercell of GaAs. The VBM is completely delocalized and looks very much like the VBM state of pure GaAs except for a region near boron impurity where the wave function is extended towards the boron atom. This distortion is precisely what one would expect based on the strong coupling of boron $p$ and arsenic $p$ states at the VBM in BAs, as discussed in Secs. III B and III D. In contrast to the VBM, the CBM state shows a significant localization around the boron atom. The CBM shows long-range delocalization, but the majority of the wave function is concentrated near the boron atom. For the second lowest conduction-band state $(\mathrm{CBM}+1)$, the situation is similar except the wave function is concentrated around a small number of gallium atoms as well. The dual character of the conduction-band states (extended at long range but concentrated around the boron atoms in the short range) indicates that the states are resonant in the conduction band and are not localized states inside the gap. The conduction-band states could be considered as boronperturbed bulk GaAs conduction-band states.

The second column of Fig. 14 shows the same states (VBM, CBM, CBM+1) for a fourth neighbor pair of boron atoms inside a supercell of GaAs. A statistically random distribution of boron atoms would result, among others, in pairs. In the case of $\mathrm{GaAs}_{1-x} \mathrm{~N}_{x}$ alloys, the presence of nitrogen pairs can result in localized impurity states inside the gap. ${ }^{61}$ Calculations were performed for the five symmetrically unique pair arrangements in the 64-atom supercell as described in Table III. Qualitative features of wave-function localization were the same for all of the pairs, and only the most representative case, the fourth nearest-neighbor pair, is shown in the figure. The features of the pair states are similar to those of the isolated impurity discussed above-that is, the VBM is mainly an As-derived, delocalized state while the lower $\mathrm{CB}$ states are concentrated around the boron atoms. Again, there is some effect on the wave function very near the boron atom. The CBM state is concentrated around the boron atom in the middle of the cell and around the boron and gallium atoms on the cell edges. The next lowest conduction-band state shows similar features and the wave function is restricted primarily to the (200) plane of the cell.

The short-range "localization", effects of boron incorporation into GaAs appear to be similar to those seen in $\mathrm{GaAs}_{1-x} \mathrm{~N}_{x}$ alloys, resulting mainly in "dual character", conduction-band states that are still extended at long range but are localized around the boron atoms. However, it appears that the perturbation of boron on the near-gap states of GaAs is gentler than that of nitrogen as none of the pairs cause states inside the gap. This is another instance (as with band-gap bowing and band offsets) where boron is less "intrusive" than its first-row cousin, nitrogen.

\section{CONCLUSIONS}

This paper explored the effects of alloying a conventional Group-III-V compound, GaAs, with boron. Very little has been known, experimentally or theoretically about boride Group-III-V semiconductor alloys, but boride semiconductor alloys are generating renewed interest now that epitaxial techniques have made it somewhat easier to fabricate them. In order to understand the fundamentals of this new class of possible alloys we have studied zincblende BAs and its alloy with GaAs.

For BAs we find: (i) The bonding in BAs is much more covalent than in the rest of the Group-III-As family or other Group-III-Vs. This is due to the similar electronegativities of boron and arsenic. (ii) Consequently, BAs has a nearly symmetric, "double-hump" bond charge density, similar to silicon. The small asymmetry that does exist in the bonding charge density actually favors boron. That is, charge is drawn toward the boron atom. In this sense, boron behaves more like the anion than the cation in BAs. (iii) The band structure of BAs is more reminiscent of silicon than most other Group-III-V compounds. This is a consequence of (1) the small repulsion between bonding and antibonding $p$ states and (2) the large repulsion of the arsenic and boron $s$ states. (iv) The LDA errors in the band gaps are approximately constant in the Group-III-As series BAs, AlAs, GaAs, and InAs. (v) The band offsets of BAs/GaAs and BAs/AlAs are unexpected. The VBM of BAs is $0.39 \mathrm{eV}$ higher than AlAs and only $0.19 \mathrm{eV}$ below GaAs. The main reason for this is the strong hybridization of both the cation and anion states at the VBM.

For the $\mathrm{B}_{x} \mathrm{Ga}_{1-x} \mathrm{As}$ alloys, we find that: (i) The bond angles are highly distorted near the boron sites. This accomodates the $\mathrm{Ga}-\mathrm{As}$ and $\mathrm{B}-\mathrm{As}$ bonds to stay close to their ideal bulk values. As a result, the bond-length distribution in a random alloy exhibits the typical bimodal behavior where each mode is near the individual ideal bulk values. (ii) The bowing parameter of $\mathrm{B}_{x} \mathrm{Ga}_{1-x} \mathrm{As}$ for low concentrations of boron is $3.5 \mathrm{eV}$, much smaller than for $\mathrm{GaAs}_{1-x} \mathrm{~N}_{x}$ alloys in the same composition range. Consequently, unlike GaN, alloying GaAs with small amounts $(<10 \%)$ of BAs increases the gap. (iii) The enthalpies of mixing indicate that the bulk solubility of boron in GaAs may be significantly higher than nitrogen in GaAs, possibly increasing the composition range over which boride alloys may be formed. (iv) The wave function of the VBM is completely delocalized and retains the character of pure GaAs. The lower conduction-band states exhibit a "semilocalized" behavior-i.e., the states are strongly concentrated around the boron atoms, but far away they are extended states.

In summary, the behavior of $\mathrm{B}_{x} \mathrm{Ga}_{1-x} \mathrm{As}$ alloys is qualitatively different from $\mathrm{GaAs}_{1-x} \mathrm{~N}_{x}$ alloys. The perturbation of boron in GaAs is much 'gentler' on most features of the electronic structure of GaAs than is nitrogen. If the same behavior is manifest for other boride Group-III-V alloys, boron offers a new class of Group-III-V alloys to be explored for interesting behavior and possible device applications. Boron may also play an important role in alloys where 
it could be used as a relatively benign component which is added to lattice match the alloy to a given substrate.

\section{ACKNOWLEDGMENTS}

This work was supported by DOE-SC-BES-DMS under Contract No. DE-AC36-99-GO10337. We gratefully ac- knowledge S.-H. Wei for many helpful discussions regarding the LAPW method and a critical reading of the manuscript, and J. F. Geisz for useful discussions regarding the experimental data and techniques as well as a critical reading of the manuscript.
${ }^{1}$ R. Addinall, R.C. Newman, Y. Okada, and F. Orito, Semicond. Sci. Technol. 7, 1306 (1992).

${ }^{2}$ R. C. Newman, Semiconductors and Semimetals (Academic, New York, 1993), Vol. 38.

${ }^{3}$ J. Maguire, R.C. Newman, I. Grant, D. Rumsby, and R.M. Ware, J. Phys. D 18, 2029 (1985).

${ }^{4}$ J. Woodhead, R.C. Newman, I. Grant, D. Rumsby, and R.M. Ware, J. Phys. C 16, 5523 (1983).

${ }^{5}$ G.A. Gledhill, R.C. Newman, and J. Woodhead, J. Phys. C 17, L301 (1984).

${ }^{6}$ S.B. Zhang and D.J. Chadi, Phys. Rev. Lett. 64, 1789 (1990).

${ }^{7}$ M.A. Tischler, P.M. Mooney, B.D. Parker, F. Cardone, and M.S. Goorsky, J. Appl. Phys. 71, 984 (1992).

${ }^{8}$ W.E. Hoke, P.J. Lemonias, D.G. Weir, and H.T. Hendricks, J. Vac. Sci. Technol. B 11, 902 (1992); S.K. Brierley, H.T. Hendricks, W.E. Hoke, P.J. Lemonias, and D.G. Weir, Appl. Phys. Lett. 63, 812 (1993).

${ }^{9}$ L.B. Ta, H.M. Hobgood, and R.N. Thomas, Appl. Phys. Lett. 41, 1091 (1982).

${ }^{10}$ S.M. Ku, J. Electrochem. Soc. 113, 813 (1966).

${ }^{11}$ S. Sakai, Y. Ueta, and Y. Terauchi, Jpn. J. Appl. Phys., Part 1 32, 4413 (1993).

${ }^{12}$ V.G. Vorob'ev, Z.S. Medvedeva, and V.V. Sobolev, Izv. Akad. Nauk SSSR, Neorg. Mater. [Inorg. Mater. (Transl. of Neorg. Mater.) 3, 959 (1967)].

${ }^{13}$ T.L. Chu and A.E. Hyslop, J. Appl. Phys. 43, 276 (1972); J. Electrochem. Soc. 121, 412 (1974).

${ }^{14}$ A.J. Perri, S. LaPlaca, and B. Post, Acta Crystallogr. 11, 310 (1958).

${ }^{15}$ F.V. Williams and R.A. Ruehrwein, J. Am. Chem. Soc. 82, 1330 (1960).

${ }^{16}$ H.M. Manasevit, W.B. Hewitt, A.J. Nelson, and A.R. Mason, J. Electrochem. Soc. 136, 3070 (1989).

${ }^{17}$ M. L. Timmons, Final Technical Report for Very High Efficiency Solar Cells, Department of Defense, Office of Contracts, Contract No. NRO-95-C-3001, 1998.

${ }^{18}$ J.F. Geisz, D.J. Friedman, J.M. Olson, Sarah R. Kurtz, R.C. Reedy, A.B. Swartzlander, B.M. Keyes, and A.G. Norman, Appl. Phys. Lett. 76, 1443 (2000).

${ }^{19}$ D.J. Stukel, Phys. Rev. B 1, 3458 (1970).

${ }^{20}$ R.M. Wentzcovitch and M.L. Cohen, J. Phys. C 19, 6791 (1986).

${ }^{21}$ R.M. Wentzcovitch, M.L. Cohen, and P.K. Lam, Phys. Rev. B 36, 6058 (1987).

${ }^{22}$ C. Prasad and M. Sahay, Phys. Status Solidi B 154, 201 (1989).

${ }^{23}$ M. Ferhat, A. Zaoui, M. Certier, and H. Aourag, Physica B 252, 229 (1998).

${ }^{24}$ M.P. Surh, S.G. Louie, and M.L. Cohen, Phys. Rev. B 43, 9126 (1991).

${ }^{25}$ F. Benkabou, C. Chikr.Z, H. Aourag, P.J. Becker, and M. Certier, Phys. Lett. A 252, 71 (1999).
${ }^{26}$ B. Bouhafs, H. Aourag, M. Ferhat, and M. Certier, J. Phys.: Condens. Matter 11, 5781 (1999).

${ }^{27}$ T. Mattila, S.-H. Wei, and A. Zunger, Phys. Rev. B 60, R11 245 (1999).

${ }^{28}$ L. Bellaiche, T. Mattila, S.-H. Wei, and A. Zunger, Phys. Rev. B 74, 1842 (1999).

${ }^{29}$ S.-H. Wei and A. Zunger, Phys. Rev. B 43, 1662 (1991); J. Appl. Phys. 78, 3846 (1995); Phys. Rev. Lett. 76, 664 (1996).

${ }^{30}$ P. Hohenberg and W. Kohn, Phys. Rev. B 136, B864 (1964); W. Kohn and L.J. Sham, ibid. 140, A1133 (1965).

${ }^{31}$ S.-H Wei and H. Krakauer, Phys. Rev. Lett. 55, 1200 (1985), and references therein; D. J. Singh, Planewaves, Pseudopotential and the LAPW Method (Kluwer Academic, Boston, 1994), and references therein.

${ }^{32}$ D.J. Singh, Phys. Rev. B 43, 6388 (1991).

${ }^{33}$ P. Blaha, K. Schwarz, and J. Luitz, WIEN97, Vienna University of Technology, Vienna, 1997; Updated version of P. Blaha, K. Schwarz, P. Sorantin, and S.B. Trickey, Comput. Phys. Commun. 59, 399 (1990).

${ }^{34}$ J.P. Perdew and Y. Wang, Phys. Rev. B 45, 13244 (1992).

${ }^{35}$ H.J. Monkhorst and J.D. Pack, Phys. Rev. B 13, 5188 (1976).

${ }^{36}$ S. Froyen, Phys. Rev. B 39, 3168 (1989).

${ }^{37}$ Tetrahedral radii are (in angstroms): boron 0.853 , aluminum 1.230, gallium and arsenic 1.225, and indium 1.405. J. C. Phillips, Bonds and Bands in Semiconductors (Academic Press, New York, 1973), p. 22.

${ }^{38}$ S.P. Kowalczyk, J.T. Cheung, E.A. Kraut, and R.W. Grant, Phys. Rev. Lett. 56, 1605 (1986); T.M. Duc, C. Hsu, and J.P. Faurie, ibid. 58, 1127 (1987).

${ }^{39}$ A. Zunger, S.-H. Wei, L.G. Ferreira, and J.E. Bernard, Phys. Rev. Lett. 65, 353 (1990); S.-H. Wei, L.G. Ferreira, J.E. Bernard, and A. Zunger, Phys. Rev. B 42, 9622 (1990).

${ }^{40}$ P.N. Keating, Phys. Rev. 145, 637 (1966); R.M. Martin, Phys. Rev. B 1, 4005 (1970).

${ }^{41}$ A.J. Williamson, L.W. Wang, and A. Zunger, cond-mat/0003055, preprint (unpublished).

${ }^{42}$ According to Phillip's scale of electronegativity (Ref. 37, p. 54), boron has a slightly higher electronegativity than arsenic. According to Pauling's scale of electronegativities, the electronegativities of boron and arsenic are the same.

${ }^{43}$ W. A. Harrison, Electronic Structure and the Properties of Solids (Freeman, San Francisco, 1980).

${ }^{44}$ S.-H. Wei and A. Zunger, Phys. Rev. B 57, 8983 (1998).

${ }^{45}$ Semiconductors. Physics of Group IV and III-V Compounds, edited by O. Madelung, M. Shulz, and H. Weiss, LandoltBörnstein, New Series, Group III, Vol. 17, Part A (SpringerVerlag, Berlin, 1982); Intrinsic Properties of Group IV Elements and III-V, II-VI, and I-VII Compounds, edited by O. Madelung, M. Shulz, and H. Weiss, Landolt-Börnstein, New Series, Group III, Vol. 22, Part A (Springer-Verlag, Berlin, 1987). 
${ }^{46}$ C.S. Wang and B.M. Klein, Phys. Rev. B 24, 3393 (1981).

${ }^{47}$ Z.W. Lu and A. Zunger, Phys. Rev. B 47, 9385 (1993).

${ }^{48}$ S.-H. Wei and A. Zunger, Appl. Phys. Lett. 72, 2011 (1998).

${ }^{49}$ S.-H. Wei and A. Zunger, Phys. Rev. Lett. 59, 144 (1987).

${ }^{50}$ J.F. Geisz, D.J. Friedman, Sarah R. Kurtz, and B.M. Keyes, J. Cryst. Growth 195, 401 (1998).

${ }^{51}$ R. Magri, S. Froyen, and A. Zunger, Phys. Rev. B 44, 7947 (1991)

${ }^{52}$ J.L. Martins and A. Zunger, Phys. Rev. B 30, 6217 (1984).

${ }^{53}$ J.C. Mikkelsen and J.B. Boyce, Phys. Rev. Lett. 49, 1412 (1982); Phys. Rev. B 28, 7130 (1983); J.B. Boyce and J.C. Mikkelsen, ibid. 31, 6903 (1985).

${ }^{54}$ Landolt-Bornstein: Numerical Data and Functional Relationships in Science and Technology, edited by O. Madelung, M. Schulz, and H. Weiss (Springer, Berlin, 1982), Vol. 17a.

55 J.E. Bernard and A. Zunger, Phys. Rev. B 36, 3199 (1987).

${ }^{56}$ L. Bellaiche, S.-H. Wei, and A. Zunger, Phys. Rev. B 56, 10233 (1997).

${ }^{57}$ J. F. Geisz (private communication).

${ }^{58}$ R.G. Dandrea, J.E. Bernard, S.-H. Wei, and A. Zunger, Phys. Rev. Lett. 64, 36 (1990).

${ }^{59}$ A. Zunger and S. Mahajan, in Handbook of Semiconductors, 2nd ed. (Elsevier, Amsterdam, 1994), Vol. 3, pp. 1399-1513.

${ }^{60}$ S.B. Zhang and Alex Zunger, Appl. Phys. Lett. 71, 677 (1997).

${ }^{61}$ X. Liu, M.-E. Pistol, L. Samuelson, S. Schwetlick, and W. Seifert, Appl. Phys. Lett. 56, 1451 (1990). 